\title{
Widespread QSO-driven outflows in the early Universe ${ }^{\star}$
}

\author{
M. Bischetti ${ }^{1,2,3}$, R. Maiolino ${ }^{2,3}$, S. Carniani ${ }^{2,3}$, F. Fiore ${ }^{4}$, E. Piconcelli ${ }^{1}$, and A. Fluetsch ${ }^{2,3}$ \\ 1 INAF - Osservatorio Astronomico di Roma, Via Frascati 33, 00078 Monte Porzio Catone, Roma, Italy \\ e-mail: manuela.bischetti@inaf.it \\ 2 Cavendish Laboratory, University of Cambridge, 19 J. J. Thomson Avenue, Cambridge CB3 0HE, UK \\ 3 Kavli Institute for Cosmology, University of Cambridge, Madingley Road, Cambridge CB3 OHA, UK \\ ${ }^{4}$ INAF - Osservatorio Astronomico di Trieste, via G.B. Tiepolo 11, 34143 Trieste, Italy
}

Received 3 June 2018 / Accepted 16 July 2019

\begin{abstract}
We present the stacking analysis of a sample of 48 quasi-stellar objects (QSOs) at $4.5<z<7.1$ detected by the Atacama Large Millimetre Array (ALMA) in the [CII] $\lambda 158 \mu \mathrm{m}$ emission line to investigate the presence and the properties of massive, cold outflows associated with broad wings in the [CII] profile. The high sensitivity reached through this analysis allows us to reveal very broad [CII] wings tracing the presence of outflows with velocities in excess of $1000 \mathrm{~km} \mathrm{~s}^{-1}$. We find that the luminosity of the broad [CII] emission increases with $L_{\mathrm{AGN}}$, while it does not significantly depend on the star formation rate of the host galaxy, indicating that the central active galactic nucleus (AGN) is the main driving mechanism of the [CII] outflows in these powerful, distant QSOs. From the stack of the ALMA cubes, we derive an average outflow spatial extent of $\sim 3.5 \mathrm{kpc}$. The average atomic neutral mass outflow rate inferred from the stack of the whole sample is $\dot{M}_{\text {out }} \sim 100 M_{\odot} \mathrm{yr}^{-1}$, while for the most luminous systems it increases to $\sim 200 M_{\odot} \mathrm{yr}^{-1}$. The associated outflow kinetic power is about $0.1 \%$ of $L_{\mathrm{AGN}}$, while the outflow momentum rate is $\sim L_{\mathrm{AGN}} / c$ or lower, suggesting that these outflows are either driven by radiation pressure onto dusty clouds or, alternatively, are driven by the nuclear wind and energy conserving but with low coupling with the interstellar medium. We discuss the implications of the resulting feedback effect on galaxy evolution in the early Universe.
\end{abstract}

Key words. galaxies: active - galaxies: high-redshift - galaxies: nuclei - quasars: emission lines - quasars: general techniques: interferometric

\section{Introduction}

The growth of super-massive black holes $(\mathrm{SMBH})$ at the centres of galaxies and the properties and evolution of the interstellar medium (ISM) in their hosts are expected to be connected (e.g. Di Matteo et al. 2005; Hopkins et al. 2008). There are in fact well-established correlations observed between the black hole mass and the physical properties of the host galaxy (Kormendy \& Ho 2013) such as the bulge mass or velocity dispersion, suggesting that the energy output of the accretion onto SMBH may be communicated to the surrounding ISM and affect star formation (SF). Indeed, active galactic nuclei (AGN) feedback onto their host galaxies is expected to proceed via kiloparsec scale, wide-angle outflows (Menci et al. 2008; Faucher-Giguère \& Quataert 2012), capable of heating and removing gas, therefore suppressing SF. AGN feedback is one of the main mechanisms invoked in cosmological simulations to prevent an excessive growth of massive galaxies and make gas-rich starburst galaxies quickly evolve to quiescence.

Growing observational evidence of massive AGN-driven outflows has been collected, involving different gas phases (ionised, atomic, and molecular) extending from sub-parsec to kiloparsec scales. While recent works, based on local AGN, use a multi-phase study of outflows to quantify their impact on the host

* The reduced continuum subtracted datacubes are only available at the CDS via anonymous ftp to cdsarc.u-strasbg.fr (130.79.128.5) or via http://cdsarc.u-strasbg.fr/viz-bin/ cat $/ \mathrm{J} / \mathrm{A}+\mathrm{A} / 630 / \mathrm{A} 59$ galaxy (e.g. Feruglio et al. 2015; Tombesi et al. 2015; Veilleux et al. 2017; Longinotti et al. 2018), at high redshift $(z \sim 1-3)$ studies of outflows are still mostly limited to the ionised phase (see Fiore et al. 2017, and references therein). There are only a few detections of fast molecular gas observed in CO high- $J$ rotational transitions (Carniani et al. 2017; Feruglio et al. 2017; Vayner et al. 2017; Brusa et al. 2018). However massive, quiescent systems and old (aged 2-3 Gyr) galaxies have already been observed at $z \sim 2-3$ (Cimatti et al. 2004; Whitaker et al. 2013; Straatman et al. 2014), indicating that a feedback mechanism must have been in place even at very early epochs, around $z \sim 5-6$.

Observations of AGN-driven outflows at high redshift have targeted the [CII] fine-structure emission line at $158 \mu \mathrm{m}$, which is generally the strongest emission line in galaxies at far infrared (FIR) wavelengths. Typically [CII] is a tracer of both the neutral atomic gas, primarily in photo-dissociated regions (PDRs), but can in part be emitted from the (partly) ionised medium (e.g. Carilli \& Walter 2013). Since PDRs are produced by the UV radiation emitted by young stars, [CII] has also been used as a tracer of SF (Maiolino et al. 2005; De Looze et al. 2011; Carniani et al. 2013, 2018).

Recently, [CII] has also been exploited to trace cold gas in galactic outflows. Indeed, broad wings [CII] emission has been observed in the hyper-luminous quasi-stellar object (QSO) $\mathrm{J} 1148+5251$ at $z \sim 6.4$ by Maiolino et al. (2012) and Cicone et al. (2015), revealing outflowing gas extended up to $\sim 30 \mathrm{kpc}$ and escaping with velocities in excess of $1000 \mathrm{~km} \mathrm{~s}^{-1}$. The Herschel Space Observatory has also enabled the detection of 
cold outflows through broad wings of the [CII] line in local active galaxies (Janssen et al. 2016).

The exploitation of the bright [CII] line at high redshift has been increasing in the last few years with the advent of the Atacama Large Millimetre Array (ALMA). In particular, the population of high- $z$ luminous QSOs with detected [CII] emission has been rapidly growing. Previous work has exploited the [CII] emission to investigate the properties of their host galaxies, such as the SFR, the dynamical mass, and the presence of merging companions (e.g. Wang et al. 2013, 2016; Venemans et al. 2016, 2017; Willott et al. 2015, 2017; Trakhtenbrot et al. 2017; Decarli et al. 2017, 2018). In none of these high-z QSOs has evidence of [CII] outflows been reported. However, most of the [CII] observations in distant QSOs are still rather short (10-20 min of on-source time), with a sensitivity generally inadequate to individually detect weak [CII] broad wings.

We collected a sample of 48 QSOs with ALMA [CII] detection to investigate the presence of outflows, as traced by weak [CII] broad wings, by performing a stacking analysis. We will show that the stacked data achieve a sensitivity that is more than an order of magnitude deeper than that reached in the previous [CII] outflow detection by Maiolino et al. (2012), Cicone et al. (2015) and enable us to reveal very broad wings tracing cold outflows associated with distant QSOs.

\section{Sample and data reduction}

We collected all [CII] observations of $z>4.5$ QSOs on the ALMA archive public as of March 2018 and selected the sources with a [CII] detection significant at $\gtrsim 5 \sigma$. Specifically, we used data from ALMA projects 2011.0.00243.S (P.I. C. Willott), 2012.1.00604.S (P.I. A. Kimball), 2012.1.00676.S (P.I. C. Willott), 2012.1.00882.S (P.I. B. Venemans), 2013.1.01153.S (P.I. P. Lira), 2015.1.01115.S (P.I. F. Walter), and 2016.1.01515.S (P.I. P. Lira). Details about individual QSOs in our sample, for those which have been published, can be found in Wang et al. (2013), Willott et al. (2013, 2015, 2017), Kimball et al. (2015), Díaz-Santos et al. (2016), Venemans et al. (2016, 2017), Decarli et al. (2017, 2018), and Trakhtenbrot et al. (2017); however, we also included some archival ALMA data that has not yet been published from project 2015.1.00997.S (P.I. R. Maiolino, Carniani et al., in prep.). The assembled sample consists of the most luminous QSOs with rest-frame absolute UV magnitude $-28.5 \lesssim M_{1450 \AA} \lesssim-23.9$ mag and black hole masses $10^{8} \lesssim M_{\mathrm{BH}} \lesssim 10^{10} M_{\odot}$. As mentioned, in total we combined ALMA data for 48 QSOs, and equivalent to a total of $\sim 34 \mathrm{~h}$ of on-source observing time.

Observations involve ALMA bands 6 or 7, depending on the redshift of the individual source. The distribution of the average rms sensitivity, representative of the [CII] spectral region, and that of the size of the ALMA beam are shown in Fig. 1. Individual values are listed in Table 1. Except for few outliers, the bulk of the observations have similar rms sensitivities from $\sim 0.3$ to $\sim 0.8 \mathrm{mJy} \mathrm{beam}^{-1}$ for a $30 \mathrm{~km} \mathrm{~s}^{-1}$ channel. The angular resolutions, computed as average beam axis, range from $\sim 0.3$ to 1.2 arcsec. Data were calibrated using the CASA 4.7.2 software (McMullin et al. 2007) in manual or pipeline mode. The default phase, bandpass and flux calibrators were used unless differently indicated by the ALMA observatory. Where necessary, extra flagging and improvement in the flux calibration was done. Data cubes were produced by using the CASA task clean by using the Hogbom algorithm with no cleaning mask and a number of iterations $N_{\text {iter }}=500-1000$ according to the significance of the detection, together with a threshold of three times the sensitivity limit given

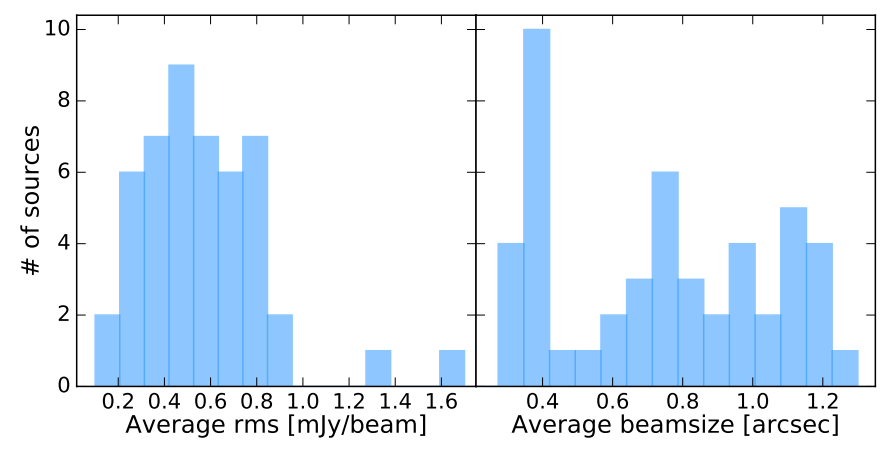

Fig. 1. Sensitivity and beamsize distributions of the ALMA [CII] observations for sampled high-z QSOs. Left panel: number of sources as a function of the mean (averaged over the spectral range covered by the stack) sensitivity for a $30 \mathrm{~km} \mathrm{~s}^{-1}$ channel. Right panel: histogram of the mean beam axis size.

by the rms. We chose a natural weighting to maximise the sensitivity of the individual observations, a common pixel size of $0.05^{\prime \prime}$ and a common spectral bin of $30 \mathrm{~km} \mathrm{~s}^{-1}$.

Continuum maps were obtained by averaging over all the four spectral windows and excluding the spectral range covered by the [CII] emission and possible [CII] broad wings. Continuum flux densities were derived by fitting a 2D Gaussian model to the ALMA maps. Furthermore, to model the continuum emission we combined the two adjacent spectral windows of the sideband containing the [CII] line to increase the available spectral range, for a total of $\sim 3.7 \mathrm{GHz}$. We did not consider the two additional spectral windows in the sideband not including [CII] because of the large spectral separation $(\sim 15 \mathrm{GHz}$ in the observed frame). The expected intrinsic differences in the QSO continuum flux $(\sim 15-20 \%)$ among this large spectral range and, mainly, the systematics in the relative calibration of distant spectral windows may affect the detection of broad wings. We thus fitted a zeroth order continuum model in the UV plane to all the available channels (of the spectral windows adjacent to [CII] where the QSO continuum variation is expected to be $<1 \%$ ) with a velocity $|v|>1500 \mathrm{~km} \mathrm{~s}^{-1}$ with respect to the centroid of the (core) [CII] emission. This choice represents a trade-off between maximising the number of channels $(\sim 1 / 4$ of each spectral window) available to the fit and avoiding spectral regions where broad [CII] wings might be present. Moreover, spectral regions corresponding to an atmospheric transmission $<0.5$ for a $1 \mathrm{~mm}$ precipitable water vapour were excluded from the fit. We verified that modelling the continuum emission with a first order polynomial did not significantly affect our results, given the limited frequency range covered by our stack.

To determine the properties of the host galaxy emission, we extracted the continuum-subtracted [CII] spectra from a region with an area of four beams (see Sect. 3). The line parameters describing the [CII] core emission were derived by fitting each spectrum with one Gaussian component model. Specifically, redshifts $\left(z_{[\mathrm{CII}]}\right)$ were derived from the centroid of the best-fit [CII] model (see Table 1).

The main properties of our high- $z$ QSOs sample are shown in Fig. 2 and listed in Table 1. The QSOs in the sample are distributed in two main redshift bins, a first group at $4.5<z<5$ and a second, higher- $z$ group at $z \gtrsim 6$. The bulk of the sample is characterised by a luminosity of the [CII] core emission in the range $\log \left(L_{[\mathrm{CII}]} / L_{\odot}\right) \sim 9.0-9.5$ and [CII] line profiles with a full width at half maximum $\left(F W H M_{[\mathrm{CII}]}^{\text {core }}\right)$ in the range between 300 and $500 \mathrm{~km} \mathrm{~s}^{-1}$. We computed the FIR luminosity by using an Mrk231-like template (Polletta et al. 2007) normalised to the 
Table 1. Main information about the ALMA observations and source properties of the QSOs in our sample.

\begin{tabular}{|c|c|c|c|c|c|c|c|c|c|c|}
\hline Source ID & $\begin{array}{c}z_{[\mathrm{CII}]}{ }^{(*)} \\
(2)\end{array}$ & $\begin{array}{l}\text { Beam } \\
\text { [arcsec] } \\
\text { (3) }\end{array}$ & $\begin{array}{c}\text { Cont rms } \\
{\left[\begin{array}{c}\left.\mathrm{mJy}_{\text {beam }}^{-1}\right] \\
\text { (4) }\end{array}\right.}\end{array}$ & $\begin{array}{c}{[\mathrm{CII}] \mathrm{rms}} \\
{\left[\mathrm{mJy} \mathrm{beam}^{-1}\right]} \\
(5)\end{array}$ & $\begin{array}{c}f_{\text {cont }} \\
{\left[\mathrm{mJy} \mathrm{beam}^{-1}\right]} \\
(6)\end{array}$ & $\begin{array}{c}L_{[\mathrm{CIII}}^{\text {core }} \\
{\left[10^{9} L_{\odot}\right]} \\
(7)\end{array}$ & $\begin{array}{c}F W H M_{[\mathrm{CII}]}^{\text {core }} \\
{\left[\mathrm{km} \mathrm{s}^{-1}\right]} \\
(8)\end{array}$ & $\begin{array}{c}\log \left(L_{\mathrm{FIR}} / L_{\odot}\right) \\
(9)\end{array}$ & $\begin{array}{c}\log \left(L_{\mathrm{AGN}} / \operatorname{erg~s}^{-1}\right)^{(* *)} \\
(10)\end{array}$ & Stack \\
\hline PJ007+04 & 6.001 & $0.47 \times 0.69$ & 0.05 & 0.58 & $1.87 \pm 0.04$ & $1.30 \pm 0.15$ & $365 \pm 45$ & 12.89 & 46.77 & $\mathrm{BF}$ \\
\hline PJ009-10 & 6.003 & $0.45 \times 0.66$ & 0.05 & 0.49 & $2.43 \pm 0.12$ & $2.28 \pm 0.15$ & $290 \pm 35$ & 12.98 & 46.73 & $\mathrm{AF}$ \\
\hline J0055+0146 & 6.005 & $0.60 \times 0.72$ & 0.03 & 0.30 & $0.22 \pm 0.02$ & $0.60 \pm 0.07$ & $330 \pm 40$ & 11.80 & 46.04 & $\mathrm{AE}$ \\
\hline J0109-3047 & 6.790 & $0.51 \times 0.80$ & 0.05 & 0.84 & $0.58 \pm 0.04$ & $1.64 \pm 0.18$ & $310 \pm 40$ & 12.30 & 46.37 & $\mathrm{AE}$ \\
\hline J0129-0035 & 5.779 & $0.36 \times 0.45$ & 0.02 & 0.24 & $3.04 \pm 0.05$ & $1.73 \pm 0.04$ & $200 \pm 30$ & 12.91 & 45.67 & $\mathrm{AF}$ \\
\hline J0142-3327 & 6.337 & $0.75 \times 0.87$ & 0.04 & 0.61 & $1.70 \pm 0.06$ & $2.94 \pm 0.16$ & $300 \pm 30$ & 12.71 & 47.24 & $\mathrm{BF}$ \\
\hline J0210-0456 & 6.433 & $0.61 \times 0.90$ & 0.03 & 0.29 & $0.16 \pm 0.03$ & $0.37 \pm 0.04$ & $185 \pm 35$ & 11.70 & 45.93 & $\mathrm{AE}$ \\
\hline J0305-3150 & 6.615 & $0.51 \times 0.72$ & 0.03 & 0.37 & $3.20 \pm 0.05$ & $2.34 \pm 0.09$ & $215 \pm 30$ & 13.02 & 46.59 & $\mathrm{AF}$ \\
\hline J0331-0741 & 4.737 & $0.31 \times 0.40$ & 0.05 & 0.45 & $3.75 \pm 0.07$ & $2.84 \pm 0.11$ & $475 \pm 35$ & 12.84 & 47.39 & DF \\
\hline PJ065-26 & 6.187 & $0.87 \times 1.11$ & 0.05 & 0.76 & $1.05 \pm 0.07$ & $1.94 \pm 0.19$ & $405 \pm 40$ & 12.48 & 47.01 & $\mathrm{DE}$ \\
\hline PJ065-19 & 6.125 & $0.75 \times 1.09$ & 0.04 & 1.32 & $0.42 \pm 0.04$ & $1.80 \pm 0.40$ & $315 \pm 60$ & 12.11 & 46.76 & $\mathrm{BE}$ \\
\hline J0454-4448 & 6.058 & $0.80 \times 1.18$ & 0.04 & 0.63 & $0.68 \pm 0.05$ & $0.62 \pm 0.10$ & $360 \pm 70$ & 12.30 & 46.68 & $\mathrm{AE}$ \\
\hline $\mathrm{J} 0807+1328$ & 4.879 & $0.25 \times 0.40$ & 0.03 & 0.67 & $6.64 \pm 0.13$ & $2.44 \pm 0.19$ & $435 \pm 38$ & 13.14 & 47.07 & DF \\
\hline $\mathrm{J} 0842+1218$ & 6.076 & $1.14 \times 1.27$ & 0.05 & 0.77 & $0.57 \pm 0.04$ & $1.62 \pm 0.22$ & $480 \pm 55$ & 12.24 & 46.88 & $\mathrm{DE}$ \\
\hline $\mathrm{J} 0923+0247$ & 4.655 & $0.29 \times 0.51$ & 0.04 & 0.30 & $2.94 \pm 0.08$ & $2.55 \pm 0.09$ & $325 \pm 30$ & 12.76 & 46.96 & $\mathrm{BF}$ \\
\hline $\mathrm{J} 0935+0801$ & 4.682 & $0.29 \times 0.55$ & 0.04 & 0.29 & $1.39 \pm 0.05$ & $0.70 \pm 0.07$ & $385 \pm 40$ & 12.48 & 47.25 & $\mathrm{BE}$ \\
\hline $\mathrm{J} 1017+0327$ & 4.949 & $0.30 \times 0.38$ & 0.03 & 0.32 & $1.23 \pm 0.07$ & $1.02 \pm 0.05$ & $270 \pm 30$ & 12.42 & 46.27 & $\mathrm{AE}$ \\
\hline PJ159-02 & 6.381 & $0.99 \times 1.27$ & 0.04 & 0.55 & $0.60 \pm 0.05$ & $1.24 \pm 0.15$ & $385 \pm 45$ & 12.27 & 46.83 & $\mathrm{BE}$ \\
\hline $\mathrm{J} 1044-0125$ & 5.785 & $0.66 \times 0.72$ & 0.02 & 0.29 & $3.07 \pm 0.03$ & $1.62 \pm 0.08$ & $470 \pm 35$ & 12.92 & 47.07 & DF \\
\hline $\mathrm{J} 1048-0109$ & 6.676 & $1.00 \times 1.43$ & 0.03 & 0.51 & $2.57 \pm 0.03$ & $2.42 \pm 0.13$ & $350 \pm 35$ & 12.94 & 46.51 & $\mathrm{AF}$ \\
\hline PJ167-13 & 6.515 & $0.98 \times 1.27$ & 0.04 & 0.51 & $0.69 \pm 0.04$ & $3.15 \pm 0.19$ & $480 \pm 35$ & 12.35 & 46.36 & $\mathrm{CE}$ \\
\hline $\mathrm{J} 1120+0641$ & 7.086 & $0.29 \times 0.32$ & 0.01 & 0.13 & $0.40 \pm 0.02$ & $0.69 \pm 0.05$ & $540 \pm 40$ & 12.19 & 46.77 & $\mathrm{DE}$ \\
\hline $\mathrm{J} 1152+0055$ & 6.365 & $1.02 \times 1.36$ & 0.04 & 0.70 & $0.50 \pm 0.06$ & $0.51 \pm 0.10$ & $140 \pm 50$ & 12.23 & 46.17 & $\mathrm{AE}$ \\
\hline $\mathrm{J} 1207+0630$ & 6.037 & $0.89 \times 1.63$ & 0.06 & 0.90 & $0.56 \pm 0.04$ & $1.16 \pm 0.18$ & $490 \pm 95$ & 12.20 & 46.77 & $\mathrm{DE}$ \\
\hline PJ183+05 & 6.439 & $1.06 \times 1.24$ & 0.04 & 0.59 & $4.62 \pm 0.05$ & $6.02 \pm 0.19$ & $370 \pm 30$ & 13.17 & 46.93 & $\mathrm{BF}$ \\
\hline $\mathrm{J} 1306+0356$ & 6.033 & $0.98 \times 1.17$ & 0.05 & 0.74 & $1.20 \pm 0.05$ & $1.87 \pm 0.17$ & $265 \pm 35$ & 12.53 & 46.84 & $\mathrm{BE}$ \\
\hline $\mathrm{J} 1319+0950$ & 6.132 & $1.10 \times 1.26$ & 0.03 & 0.43 & $5.00 \pm 0.05$ & $3.85 \pm 0.18$ & $520 \pm 35$ & 13.17 & 46.93 & DF \\
\hline $\mathrm{J} 1321+0038$ & 4.722 & $0.34 \times 0.39$ & 0.02 & 0.19 & $1.49 \pm 0.04$ & $1.19 \pm 0.06$ & $560 \pm 35$ & 12.47 & 46.70 & $\mathrm{CE}$ \\
\hline $\mathrm{J} 1328-0224$ & 4.646 & $0.31 \times 0.48$ & 0.04 & 0.37 & $1.58 \pm 0.04$ & $1.56 \pm 0.06$ & $300 \pm 30$ & 12.49 & 47.05 & $\mathrm{BE}$ \\
\hline $\mathrm{J} 1341+0141$ & 4.700 & $0.30 \times 0.38$ & 0.06 & 0.45 & $17.74 \pm 0.33$ & $3.06 \pm 0.15$ & $435 \pm 35$ & 13.55 & 47.50 & DF \\
\hline $\mathrm{J} 1404+0314$ & 4.924 & $0.34 \times 0.40$ & 0.05 & 0.58 & $10.98 \pm 0.20$ & $3.14 \pm 0.15$ & $515 \pm 35$ & 13.37 & 47.02 & DF \\
\hline PJ217-16 & 6.149 & $0.92 \times 1.19$ & 0.05 & 0.71 & $0.40 \pm 0.02$ & $0.89 \pm 0.17$ & $510 \pm 70$ & 12.14 & 46.89 & DE \\
\hline $\mathrm{J} 1433+0227$ & 4.727 & $0.34 \times 0.44$ & 0.05 & 0.43 & $7.69 \pm 0.13$ & $2.52 \pm 0.08$ & $415 \pm 30$ & 13.19 & 47.37 & DF \\
\hline $\mathrm{J} 1509-1749$ & 6.122 & $0.92 \times 1.43$ & 0.04 & 0.67 & $1.34 \pm 0.04$ & $1.72 \pm 0.20$ & $615 \pm 75$ & 12.59 & 46.9 & DE \\
\hline $\mathrm{J} 1511+0408$ & 4.679 & $0.31 \times 0.53$ & 0.06 & 0.46 & $10.08 \pm 0.19$ & $2.78 \pm 0.18$ & $580 \pm 45$ & 13.30 & 47.25 & DF \\
\hline PJ231-20 & 6.587 & $0.94 \times 1.29$ & 0.04 & 0.66 & $3.80 \pm 0.10$ & $2.97 \pm 0.21$ & $410 \pm 35$ & 13.09 & 46.99 & DF \\
\hline $\mathrm{J} 1554+1937$ & 4.627 & $0.74 \times 1.26$ & 0.16 & 1.67 & $11.98 \pm 0.42$ & $6.86 \pm 0.43$ & $800 \pm 45$ & 13.37 & 47.70 & DF \\
\hline PJ308-21 & 6.234 & $0.68 \times 0.89$ & 0.03 & 0.54 & $1.12 \pm 0.08$ & $2.17 \pm 0.18$ & $575 \pm 45$ & 12.53 & 46.65 & $\mathrm{CE}$ \\
\hline $\mathrm{J} 2054-0005$ & 6.039 & $0.73 \times 0.76$ & 0.02 & 0.40 & $2.89 \pm 0.04$ & $2.46 \pm 0.07$ & $230 \pm 30$ & 12.92 & 46.60 & $\mathrm{AF}$ \\
\hline $\mathrm{J} 2100-1715$ & 6.082 & $0.66 \times 0.78$ & 0.05 & 0.60 & $0.46 \pm 0.02$ & $1.27 \pm 0.17$ & $390 \pm 60$ & 12.22 & 46.33 & $\mathrm{AE}$ \\
\hline $\mathrm{J} 2229+1457$ & 6.151 & $0.70 \times 0.80$ & 0.03 & 0.42 & $0.14 \pm 0.02$ & $0.34 \pm 0.06$ & $240 \pm 50$ & 11.62 & 46.03 & $\mathrm{AE}$ \\
\hline $\mathrm{J} 2244+1346$ & 4.661 & $0.33 \times 0.40$ & 0.03 & 0.31 & $3.26 \pm 0.05$ & $1.74 \pm 0.04$ & $270 \pm 30$ & 12.80 & 46.58 & $\mathrm{AF}$ \\
\hline W2246-0526 & 4.601 & $0.35 \times 0.37$ & 0.05 & 0.52 & $7.18 \pm 0.12$ & $6.12 \pm 0.19$ & $740 \pm 35$ & 13.14 & 48.12 & DF \\
\hline $\mathrm{J} 2310+1855$ & 6.002 & $0.79 \times 1.18$ & 0.04 & 0.41 & $7.62 \pm 0.12$ & $5.74 \pm 0.15$ & $405 \pm 30$ & 13.34 & 47.23 & DF \\
\hline $\mathrm{J} 2318-3029$ & 6.148 & $0.75 \times 0.87$ & 0.05 & 0.82 & $2.87 \pm 0.05$ & $1.73 \pm 0.18$ & $275 \pm 35$ & 12.93 & 46.60 & $\mathrm{AF}$ \\
\hline $\mathrm{J} 2318-3113$ & 6.444 & $0.79 \times 0.89$ & 0.06 & 0.92 & $0.32 \pm 0.04$ & $1.26 \pm 0.20$ & $305 \pm 55$ & 12.00 & 46.56 & $\mathrm{AE}$ \\
\hline $\mathrm{J} 2348-3054$ & 6.902 & $0.62 \times 0.82$ & 0.05 & 0.79 & $1.90 \pm 0.05$ & $1.52 \pm 0.23$ & $455 \pm 65$ & 12.82 & 46.43 & $\mathrm{CF}$ \\
\hline PJ359-06 & 6.172 & $0.64 \times 1.14$ & 0.07 & 0.77 & $0.76 \pm 0.05$ & $1.66 \pm 0.17$ & $305 \pm 40$ & 12.46 & 46.83 & $\mathrm{BE}$ \\
\hline
\end{tabular}

Notes. Columns give the following information: (1) source ID, (2) [CII]-based redshift, (3) x, (4) continuum rms sensitivity, (5) representative rms of the [CII] spectral region for a channel width of $30 \mathrm{~km} \mathrm{~s}^{-1}$, (6) continuum flux, (7) [CII] luminosity, (8) FWHM of the [CII] line, (9) FIR luminosity in the range $8-1000 \mu \mathrm{m}$ derived from the ALMA continuum flux, (10) AGN bolometric luminosity. ${ }^{(*)}$ Given the statistical error on the centroid of the best-fit Gaussian modelling the [CII] line and the systematics associated with the $30 \mathrm{~km} \mathrm{~s}^{-1}$ channel width of our ALMA spectra, the typical error on redshift is $\Delta z_{[\mathrm{CII}]}=0.001$. $^{(*)}$ We consider as error on $L_{\mathrm{AGN}}$ the 0.1 dex scatter associated with the bolometric correction by Runnoe et al. (2012).

observed continuum flux density at (rest frame) $158 \mu \mathrm{m}$. The resulting $L_{\text {FIR }}$ (see Fig. 2) span almost two orders of magnitude, that is $\log \left(L_{\mathrm{FIR}} / L_{\odot}\right)=11.6-13.4$. The AGN bolometric luminosity $\left(L_{\mathrm{AGN}}\right)$ was derived from the monochromatic luminosity at $1450 \AA$ by applying the bolometric correction from Runnoe et al. (2012). All sources in our sample are luminous and hyperluminous QSOs with $L_{\mathrm{AGN}} \gtrsim 10^{46} \mathrm{erg} \mathrm{s}^{-1}$, with an average $L_{\mathrm{AGN}}$ of $6.3 \times 10^{46} \mathrm{erg} \mathrm{s}^{-1}$.

\section{Methods}

In order to investigate the presence of high velocity wings of the [CII] emission, we performed a stacking analysis of the distant QSOs in our sample. The stacking technique has the potential to greatly increase the sensitivity of the stacked spectrum or stacked cube and, therefore, favours the detection of even modest outflows traced by weak [CII] wings. 

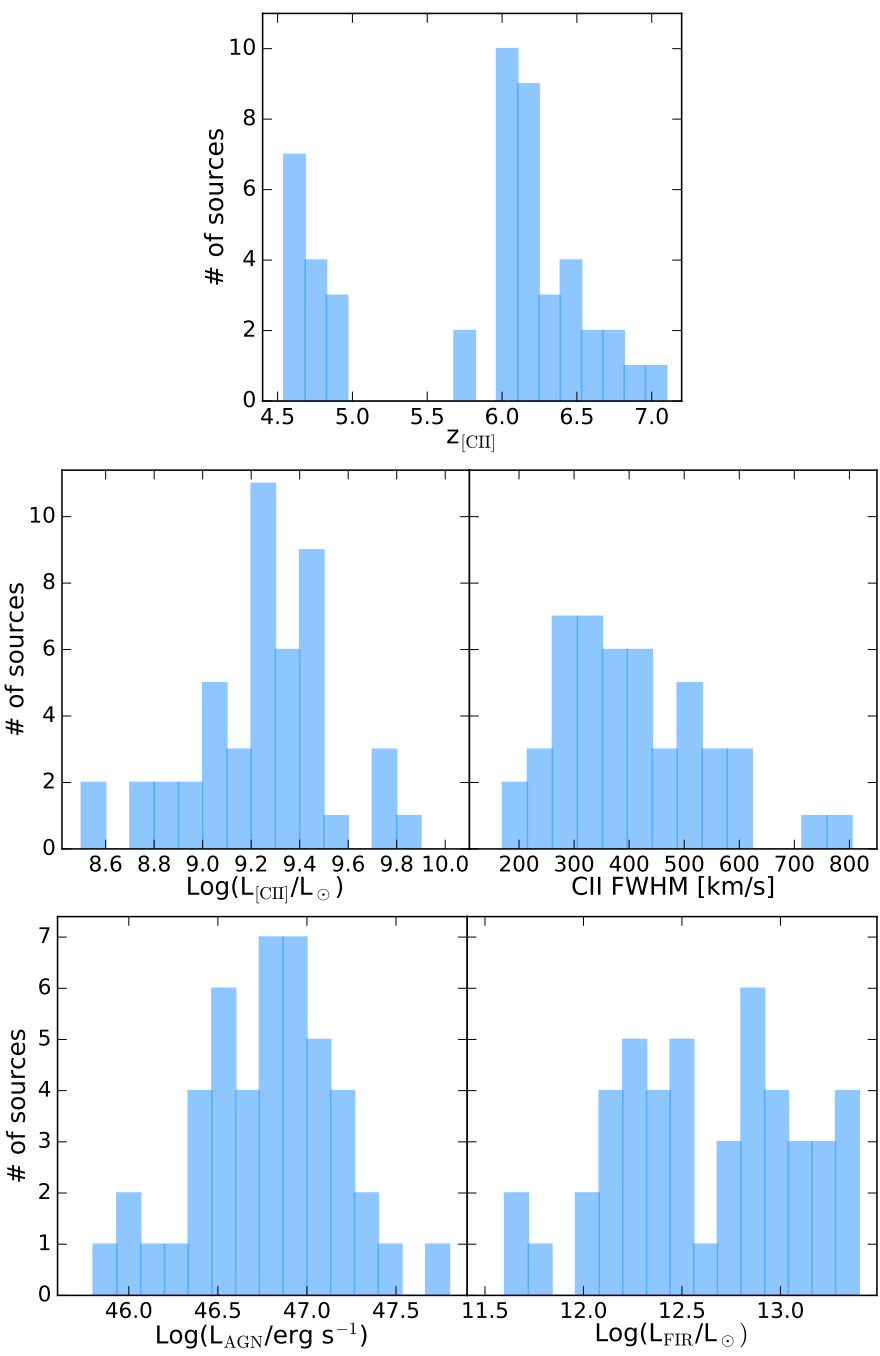

Fig. 2. Properties of high-z QSOs sample considered in this work Top panel: redshift distribution. Middle panel: luminosity and full width at half maximum (FWHM) of the [CII] core emission. Bottom panel: AGN luminosity and FIR luminosity.

As a first step, the cubes were aligned at the [CII] rest frequency $(1900.5369 \mathrm{GHz})$ according to $z_{[\mathrm{CII}]}$ and spatially centred on the peak of the QSO continuum emission. We did not include in the stack spectral regions corresponding to an atmospheric transmission $<0.5$ for a $1 \mathrm{~mm}$ precipitable water vapour. Then, we combined the data from the 48 sources in our sample according to the relation below, defining the weighted intensity $I_{k}^{\prime}$ of a generic spatial pixel $\left(x^{\prime}, y^{\prime}\right)$ in the stacked cube for each spectral channel $k$, and the relative weight $W_{\mathrm{k}}^{\prime}$ as follows (Fruchter $\&$ Hook 2002):

$W_{\mathrm{k}}^{\prime}=\sum_{j=1}^{n} w_{\mathrm{j}, \mathrm{k}}=\sum_{j=1}^{n} \frac{1}{\sigma_{\mathrm{j}, \mathrm{k}}^{2}}=\frac{1}{\sigma_{k}^{\prime 2}}$

$I_{k}^{\prime}=\frac{\sum_{j=1}^{n}\left(i_{\mathrm{j}, \mathrm{k}} \cdot w_{\mathrm{j}, \mathrm{k}}\right)}{W_{k}^{\prime}}$,

where $i_{\mathrm{j}, \mathrm{k}}$ is the intensity at the same spatial pixel $\left(x_{j}, y_{j}\right)$ and same spectral channel $k$ of source $j$, and $n=48$.

We applied a standard variance-weighted stacking, that is we used a weighting factor $w_{\mathrm{j}, \mathrm{k}}=1 / \sigma_{\mathrm{j}, \mathrm{k}}^{2}$, where $\sigma_{\mathrm{j}, \mathrm{k}}$ is the root mean square (rms) noise estimated channel by channel from cube $j$.
Furthermore, with this method we accounted for the noise variation with frequency in the spectral range covered by the ALMA [CII] spectra, that is $\sim 3.7 \mathrm{GHz}$, and considered only the contribution of sources with available spectral coverage in our weighted mean. We performed the stacking in two alternative, complementary ways: by stacking the 1D spectra extracted from the individual cubes and by stacking the $3 \mathrm{D}$ cubes into a single stacked cube.

In the first case the continuum-subtracted spectrum of each target was extracted from an elliptical aperture with same position angle of the beam, but over an area four times larger. This approach allows us to collect most of the flux from the QSO (for a point source, $294 \%$ of the flux lies within two beam axes) and limits the contamination of possible companions. The angular size of the systemic [CII] emission is comparable to the ALMA beam for most of the QSOs in our sample (e.g. Venemans et al. 2016, 2017; Decarli et al. 2018). The chosen extraction areas therefore maximise the significance of possible high-velocity [CII] wings if outflowing and systemic gas are distributed over similar scales (Cicone et al. 2014). As a drawback of our approach, emission from different physical scales may contribute to the stacked spectra. The individual spectra were stacked according to Eqs. (1) and (2).

In the second approach, the continuum-subtracted cubes of the single sources were stacked by applying Eqs. (1), (2) to each spaxel. This resulted in a stacked data cube, containing the contribution of each source to the different channels and spatial positions.

Table 2 reports the statistical uncertainties of the stacked spectrum and cube in spectral channel of $30 \mathrm{~km} \mathrm{~s}^{-1}$, which have been estimated excluding those spaxels contaminated by the QSO emission. We note that the statistical uncertainty of a spectrum extracted from an area of $\mathrm{N}$ beams was computed as $\sqrt{N}$ times the rms. Similarly, the sensitivity of a map integrated over $\mathrm{K}$ channels was derived as $\left(\sum_{i=1}^{K} \sigma_{i}^{-2}\right)^{-1 / 2}$, where $\sigma_{i}$ is the rms within each channel slice.

To ensure that the presence of broad [CII] wings in the stacked spectrum is not an artefact of the stacking procedure, we extracted individual integrated spectra from 100 "empty" positions randomly-selected within the ALMA field of view and stacked them as described above. An upper limit on the significance of the integrated flux associated with a spurious broad component can be derived by fitting the stacked noise spectra with one broad Gaussian profile with a $F W H M>500 \mathrm{~km} \mathrm{~s}^{-1}$ and centroid in the velocity range $v \in[-500,+500] \mathrm{km} \mathrm{s}^{-1}$. This resulted into an average spurious signal-to-noise ratio of $\sim 0.4$ for the stack of the total sample.

We also verified that the presence of broad [CII] wings was not associated with a few QSOs but instead a general property of our sample. For this purpose, we recomputed 1000 times the stack of the integrated spectrum on different subgroups, excluding each time a combination of five randomly selected sources ( $\sim 10 \%$ of the sample). The resulting rms variation of the [CII] wings in the velocity range $400<|v|<1500 \mathrm{~km} \mathrm{~s}^{-1}$ corresponds to $\sim 20 \%$ of the peak flux density of the broad [CII] wings presented in Sect. 4.1. The average luminosity variation of the [CII] wings is $\sim 11 \%$, with a maximum variation of $40 \%$.

The uncertainty on the continuum fitting of the individual spectra would result into a simple pedestal, as we modelled the continuum emission with a zero order. However, the fitting of the total stacked spectrum does include a continuum component which is fully consistent with zero, confirming on average a proper continuum subtraction in the individual spectra. 
Table 2. Variance-weighted properties of the stacked QSO samples and the corresponding [CII] emission properties.

\begin{tabular}{|c|c|c|c|c|c|c|c|c|}
\hline & & \multirow{2}{*}{$\begin{array}{l}\text { Whole } \\
\text { sample }\end{array}$} & \multicolumn{6}{|c|}{ Subsamples } \\
\hline & & & A & $\mathrm{B}$ & $\mathrm{C}$ & $\mathrm{D}$ & $\mathrm{E}$ & $\mathrm{F}$ \\
\hline (1) & rms $\left[\mathrm{mJy}\right.$ beam $\left.^{-1}\right]$ & 0.06 & 0.11 & 0.16 & 0.16 & 0.09 & 0.09 & 0.10 \\
\hline (2) & $L_{\mathrm{AGN}}\left[\mathrm{erg} \mathrm{s}^{-1}\right]$ & 47.0 & 46.3 & 47.1 & 46.7 & 47.2 & 46.7 & 47.1 \\
\hline (3) & $\mathrm{SFR}_{\mathrm{FIR}}\left[M_{\odot} \mathrm{yr}^{-1}\right]$ & 790 & 570 & 540 & 360 & 1270 & 260 & 1750 \\
\hline (4) & $F W H M_{[\mathrm{CII}]}^{\text {core }}\left[\mathrm{km} \mathrm{s}^{-1}\right]$ & $390 \pm 30$ & $210 \pm 30$ & $330 \pm 30$ & $600 \pm 40$ & $510 \pm 40$ & $390 \pm 30$ & $360 \pm 30$ \\
\hline (5) & $L_{[\mathrm{CII}]}^{\text {broad }}\left[10^{8} L_{\odot}\right]$ & $4.1 \pm 0.7$ & $2.5 \pm 0.8$ & $4.6 \pm 1.9$ & $3.7 \pm 1.2$ & $6.9 \pm 1.5$ & $4.2 \pm 1.1$ & $3.8 \pm 0.8$ \\
\hline (6) & $S / N_{[\mathrm{CII}]}^{\text {broad }}$ & $5.6^{(*)}, 10.2^{(* *)}, 7.2^{(* * *)}$ & $3.0,5.6,3.5$ & $2.4,7.5,3.3$ & $3.0,2.9,2.4$ & $4.6,7.0,9.8$ & $3.7,6.5,5.1$ & $4.8,7.8,5.4$ \\
\hline (7) & $F W H M_{[\mathrm{CII}]}^{\text {broad }}\left[\mathrm{km} \mathrm{s}^{-1}\right]$ & $1730 \pm 210$ & $850 \pm 160$ & $710 \pm 130$ & $2360 \pm 640$ & $1920 \pm 250$ & $2210 \pm 430$ & $1380 \pm 200$ \\
\hline (8) & $\Delta v\left[\mathrm{~km} \mathrm{~s}^{-1}\right]$ & $-90 \pm 40$ & $-110 \pm 70$ & $-70 \pm 50$ & $-10 \pm 100$ & $-180 \pm 70$ & $130 \pm 100$ & $-240 \pm 90$ \\
\hline (9) & $p_{[\mathrm{CII}]}$ & $0.05 \pm 0.01$ & $0.05 \pm 0.01$ & $0.1 \pm 0.03$ & $0.05 \pm 0.02$ & $0.07 \pm 0.01$ & $0.07 \pm 0.01$ & $0.04 \pm 0.01$ \\
\hline (10) & $f_{[\mathrm{CII}]}$ & $0.22 \pm 0.04$ & $0.18 \pm 0.06$ & $0.23 \pm 0.08$ & $0.22 \pm 0.07$ & $0.31 \pm 0.06$ & $0.39 \pm 0.09$ & $0.14 \pm 0.02$ \\
\hline
\end{tabular}

Notes. Specifically, rows give the following information: (1) rms sensitivity representative of the [CII] spectral region for a channel width of $30 \mathrm{~km} \mathrm{~s}^{-1}$, (2) average $L_{\mathrm{AGN}}$ in the (sub-)sample, (3) average FIR-based SFR, (4) FWHM of the [CII] core, (5) average luminosity of the broad [CII] wings, (6) their significance, (7) FWHM, and (8) velocity shift. (9) Peak and (10) integrated flux density ratios of the broad [CII] with respect to the core $[\mathrm{CII}]$ emission. ${ }^{(*)}$ Computed from the fit parameters errors, accounts for the uncertainty in modelling the narrow component.

${ }^{(* *)}$ Computed from the pure statistical uncertainty. ${ }^{(* *)}$ Computed as in ${ }^{(* *)}$, but excluding the central channels affected by the [CII] core.

\section{Results}

\subsection{Stacked spectrum}

The integrated spectrum resulting from the stack of all 48 QSO individual (1D) spectra in our sample is shown in Fig. 3. The stacked spectrum reveals very broad wings beneath the narrow line core, tracing fast outflows of cold gas.

We modelled the spectrum with a single Gaussian component. The $\chi^{2}$ minimisation of the fit was performed by using for each channel the weight $W_{\mathrm{k}}^{\prime}$ (see Eq. (1)) and all the model parameters were free to vary in the fit with no constrains. However, a single Gaussian model could not account for the emission at $v>500 \mathrm{~km} \mathrm{~s}^{-1}$. This can be seen in the first bottom panel of Fig. 3 as also indicated by the resulting large $\chi_{v, 1 \mathrm{G}}^{2}=8.6$. The addition of a second unconstrained Gaussian component gives a $\chi_{v}^{2}=3.7$ (see Fig. 3), that is a factor of $\gtrsim 2$ smaller, which indicates that the second, broad Gaussian component is required with very high confidence level $(>99.9 \%)$. The reduced $\chi^{2}$ is yet larger than unity, hence suggesting that the line profile might be more complex than two simple Gaussian components. Details on the fitting procedure, uncertainties and confidence ellipses associated with the parameters of the broad Gaussian modelling the [CII] wings, are reported in Appendix A. In Appendix A we also show the results from a single-Gaussian model fitting, indicating the reliability of the broad component.

The significance estimated through the fitting analysis is $5.6 \sigma$. The significance based on the simple integration of the flux associated with the broad component (and the statistical uncertainty calculated in the same spectral region) gives a significance of $\sim 10 \sigma$. This is a pure statistical significance of the broad signal, higher than the confidence obtained from the fit, as it does not take into account the uncertainties associated with the subtraction of the narrow component. However, even ignoring the central channels affected by the core, the statistical significance of the wings alone is $\sim 7 \sigma$ (see Table 2 ).

The median $\mathrm{rms}$ of the stacked spectrum is $\sim 0.06 \mathrm{mJy}$ beam $^{-1}$ (see Table 2), which is consistent with the noise expected by stacking the original spectra if the noise is Gaussian. To give an idea of the significant improvement in sensitivity obtained with the stack, the sensitivity level reached in this work is a factor of $\sim 14$ lower than that of the $\mathrm{J} 1148+5251$ observations of Cicone et al. (2015), where a massive [CII] outflow was found.

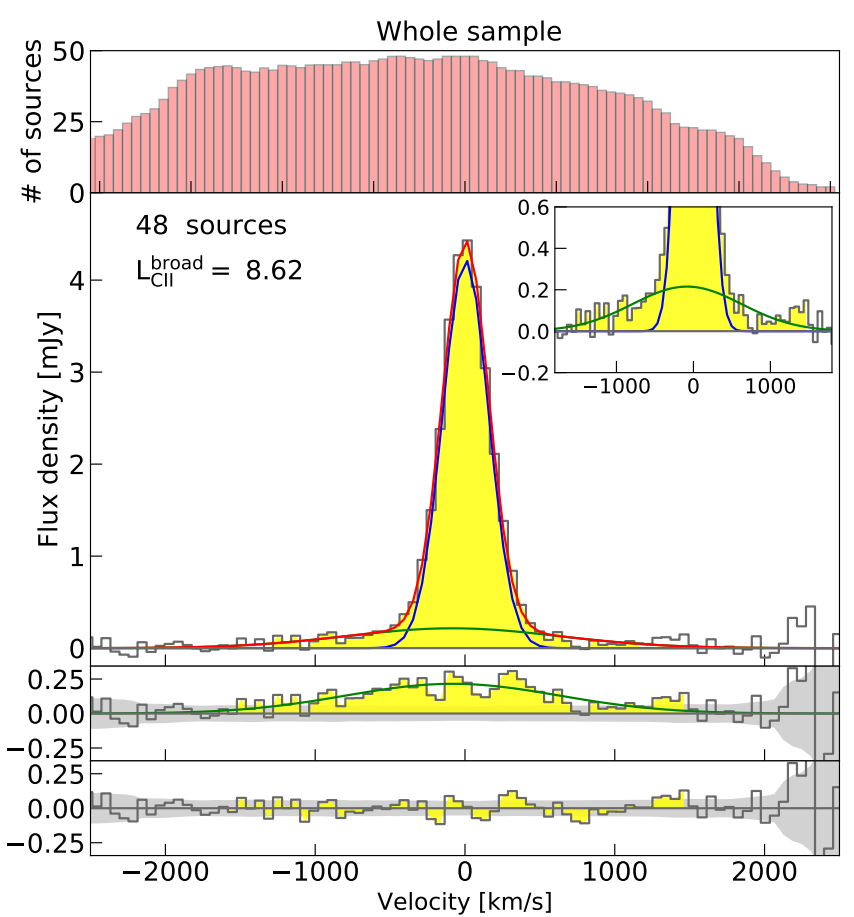

Fig. 3. Whole sample stacked integrated spectrum. First panel from top: number of sources contributing to the stack at different velocities. Second panel from top: [CII] flux density as a function of velocity, in spectral bins of $60 \mathrm{~km} \mathrm{~s}^{-1}$. The red curve represents the best-fit 2 Gaussian components model: the combination of a core component (blue) and a broad component (green) is needed to properly reproduce the data. Labels indicate the number of stacked sources and the luminosity of the broad [CII] wings. The inset shows a zoom on the broad component. Third panel from top: residuals from the subtraction of the core component (blue line in the second panel). The green curve shows the best fit broad component. Fourth panel: residuals from the two Gaussian components fitting. The $1 \sigma \mathrm{rms}$ of the spectrum is also indicated by the shaded region.

In the stacked spectrum the core emission component has a width of $F W H M_{[\mathrm{CII}]}^{\text {core }}=390 \pm 30 \mathrm{~km} \mathrm{~s}^{-1}$, while the underlying very broad component has a width of $F W H M_{[\mathrm{CII}]}^{\text {broad }}=1730 \pm$ $210 \mathrm{~km} \mathrm{~s}^{-1}$ (see Table 2). The broad wings are not symmetric, the 
blue side being much more prominent than the red side, resulting in the overall broad Gaussian used to fit the broad component being slightly blueshifted (by $\sim 90 \mathrm{~km} \mathrm{~s}^{-1}$, see Table 2) with respect to the systemic [CII] emission. This might be an artefact resulting from the asymmetric distribution of the data, with the red wing being contributed by fewer spectra than the blue wing (top panel of Fig. 3). Alternatively, at such early epochs, the host galaxies of these hyper-luminous QSOs may be similar to extreme ultra-luminous infrared galaxies (ULIRGs), which have been found to be optically thick even at far-IR and submillimetre wavelengths (Papadopoulos et al. 2010; Neri et al. 2014; Gullberg et al. 2015), which may result into absorption of the receding (redshifted) component of the outflow, even at the wavelength of [CII]. The latter interpretation is supported by the fact that, as we will show in Sect. 4.2, when we produce stacks by splitting the sample between galaxies with high and low SFR (hence high and low gas and dust content), the stack associated with low SFR (hence low dust content) does not show a blueshift of the broad component, while the sample with high SFR (hence high dust content) exhibits a large blueshift of the [CII] broad component.

The peak flux density of the broad [CII] component is about $5 \%$ of that of the core, while the integrated broad-to-narrow [CII] flux density ratio is $f_{[\mathrm{CII}]} \sim 0.22$ (see Table 2 ). In order to estimate the luminosity of the [CII] broad component representative of our sample, we computed a weighted $L_{[\mathrm{CII}]}^{\text {stack }}$ by applying Eqs. (1) and (2) to the individual narrow [CII] luminosities of our targets. We therefore derived the luminosity associated with the broad [CII] wings as $L_{[\mathrm{CII}]}^{\text {broad }}=L_{[\mathrm{CII}]}^{\text {stack }} \times f_{[\mathrm{CII}]}$.

The individual [CII] spectra contributing to the stacked spectrum are characterised by FWHM of the line core in the range $\sim 150-800 \mathrm{~km} \mathrm{~s}^{-1}$ (Table 1). The combination of line profiles with different widths may potentially result into a stacked profile similar to the combination of a narrow and a broad Gaussian curve. Although the latter could not be as broad as the wings observed in the stacked spectrum of Fig. 3, this could still contribute to $L_{[\mathrm{CII}]}^{\text {broad }}$. We quantified this contribution by stacking Gaussian curves with the same FWHM and $L_{[\mathrm{CII}]}$ distribution of the QSOs in our sample, according to Eqs. (1) and (2). In the stacked profile, we computed the [CII] emission in excess of a single Gaussian curve to be $\sim 17 \%$ of $L_{[\mathrm{CII}]}^{\mathrm{broad}}$, indicating that the effect mentioned above can only have a marginal contribution to the flux of the broad component.

\subsection{Outflow relation with QSO-galaxy properties}

In this section, we study the relation between the presence of cold outflows traced by the [CII] wings and the properties of the QSO-host galaxy system, such as AGN luminosity and SFR. Furthermore, to investigate the presence of broad [CII] wings without the shortcomings of combining [CII] profiles with significantly different widths, we separate the QSOs in two subsamples with $F W H M_{[\mathrm{CII}]}<400 \mathrm{~km} \mathrm{~s}^{-1}$ (median linewidth of the whole sample) and $F W H M_{[\mathrm{CII}]}>400 \mathrm{~km} \mathrm{~s}^{-1}$, respectively. This roughly corresponds to discriminate between less and more massive systems, given that the [CII] linewidth is a proxy of the dynamical mass of the galaxy (modulo disc inclination effects). We further separate our sample in two AGN luminosity bins: specifically $L_{\mathrm{AGN}}<10^{46.8} \mathrm{erg} \mathrm{s}^{-1}$ (median $L_{\mathrm{AGN}}$ of the whole sample) and $L_{\mathrm{AGN}}>10^{46.8} \mathrm{erg} \mathrm{s}^{-1}$. This allowed us to investigate the relation between the $[\mathrm{CII}]$ outflow strength and $L_{\mathrm{AGN}}$. For simplicity, hereafter the different subsamples will be referred to as:

$$
\begin{aligned}
& -A: F W H M_{[\mathrm{CII}]}<400 \mathrm{~km} \mathrm{~s}^{-1}, L_{\mathrm{AGN}}<10^{46.8} \mathrm{erg} \mathrm{s}^{-1} \\
& -B: F W H M_{[\mathrm{CII}]}<400 \mathrm{~km} \mathrm{~s}^{-1}, L_{\mathrm{AGN}}>10^{46.8} \mathrm{erg} \mathrm{s}^{-1} \\
& -C: F W H M_{[\mathrm{CII}]}>400 \mathrm{~km} \mathrm{~s}^{-1}, L_{\mathrm{AGN}}<10^{46.8} \mathrm{erg} \mathrm{s}^{-1} \\
& -D: F W H M_{[\mathrm{CII}]}>400 \mathrm{~km} \mathrm{~s}^{-1}, L_{\mathrm{AGN}}>10^{46.8} \mathrm{erg} \mathrm{s}^{-1}
\end{aligned}
$$

The stacked spectra for the different subsamples are shown in Fig. 4. Because of less statistics, the sensitivity improvement is modest compared to the stack of the whole sample (see Table 2) and the individual source contribution to the stacked spectrum is more evident. This is particularly evident in Fig. 4 for stacks $C$ and $D$, where the core of the stacked [CII] profile is broadened by few sources exhibiting a rotation pattern in their [CII] spectra. We fit $A$ and $B$ with a two Gaussian components model, while for stack $D$ and $E$ we use a combination of two Gaussians to account for the broadening of the [CII] core, and a third Gaussian to reproduce the [CII] wings. Similarly to Sect. 4.4, all parameters were let free to very in the fit with no constrains. The best fit models are shown in Fig. 4.

A faint broad [CII] emission component is still observed in the stacked spectrum of the subgroups, although with lower significance if compared to the whole sample stack presented in Sect. 4.1 and, in few cases, with only marginal significance (see Table 2). In sources with small FWHM of the [CII] core emission (stacks $A$ and $B$ ), wings are characterised by $F W H M_{[\mathrm{CII}]}^{\text {broad }}$ up to $\sim 850 \mathrm{~km} \mathrm{~s}^{-1}$, while broader wings with $F W H M_{[\mathrm{CII}]}^{\text {broad }} \sim 2000 \mathrm{~km} \mathrm{~s}^{-1}$ are observed in the subsamples with broader [CII] cores (stacks $C$ and $D$ ). Similarly to what we found in the whole sample stack, the peak of the broad [CII] wings is $5 \%$ to $10 \%$ of the core peak flux density, while the integrated flux of the broad component corresponds to 20-30\% of the core component. Following the same method presented in Sect. 3 for the stack of the total sample, the average signal-to-noise ratio of a spurious broad component is $\sim 0.4-0.6$.

For each subsample, $L_{[\mathrm{CII}]}^{\text {broad }}$ and $L_{\mathrm{AGN}}$ have been computed following the same method of Sect. 4.1 (see Table 2). We observe an increased $L_{[\mathrm{CII}]}^{\text {broad }}$ in the high $L_{\mathrm{AGN}}$ sources (see Fig. 4), despite the limited luminosity range spanned by the sources considered in our analysis. Fig. 5 shows indeed that the stacked $L_{[\mathrm{CII}]}^{\text {broad }}$ follow a trend with $L_{\mathrm{AGN}}$ similar to what observed by previous works in individual sources at lower redshift (e.g. Cicone et al. 2014; Fiore et al. 2017; Fluetsch et al. 2019), finding that the outflow strength correlates with the AGN luminosity. This result indicates that the observed [CII] outflows are primarily QSO-driven. Instead, we see only marginal variations of $L_{[\mathrm{CII}]}^{\text {broa }}$ with respect to the width of the line core, indicating that the dynamics of the galactic disc does not significantly affect the detectability of the broad [CII] components associated with the outflow.

An alternative driving mechanism of the fast [CII] emission could be the starburst in the QSO host galaxy through supernovae and radiation pressure. To investigate this possibility in more detail, we considered two subgroups according to their SFR, as inferred from their $L_{\mathrm{FIR}}$ (computed following Kennicutt \& Evans 2012), assuming that the bulk of the far-IR emission is associated with $\mathrm{SF}$ in the host galaxy:

- E: $\mathrm{SFR}_{\mathrm{FIR}}<600 M_{\odot} \mathrm{yr}^{-1}$

- $F: \mathrm{SFR}_{\mathrm{FIR}}>600 M_{\odot} \mathrm{yr}^{-1}$

The corresponding stacked spectra are shown in Fig. 6. It is evident that the [CII] core emission is mainly associated with SF activity, confirming that [CII] is a tracer of star formation as previously found by De Looze et al. (2014), Herrera-Camus et al. (2015) for example. The [CII] flux density of the core in stack $E$, characterised by a variance-weighted SFR of $260 M_{\odot} \mathrm{yr}^{-1}$, is in fact a factor of $\sim 3.5$ lower with respect to the highly star forming sources stacked in $F$ (whose variance-weighted 

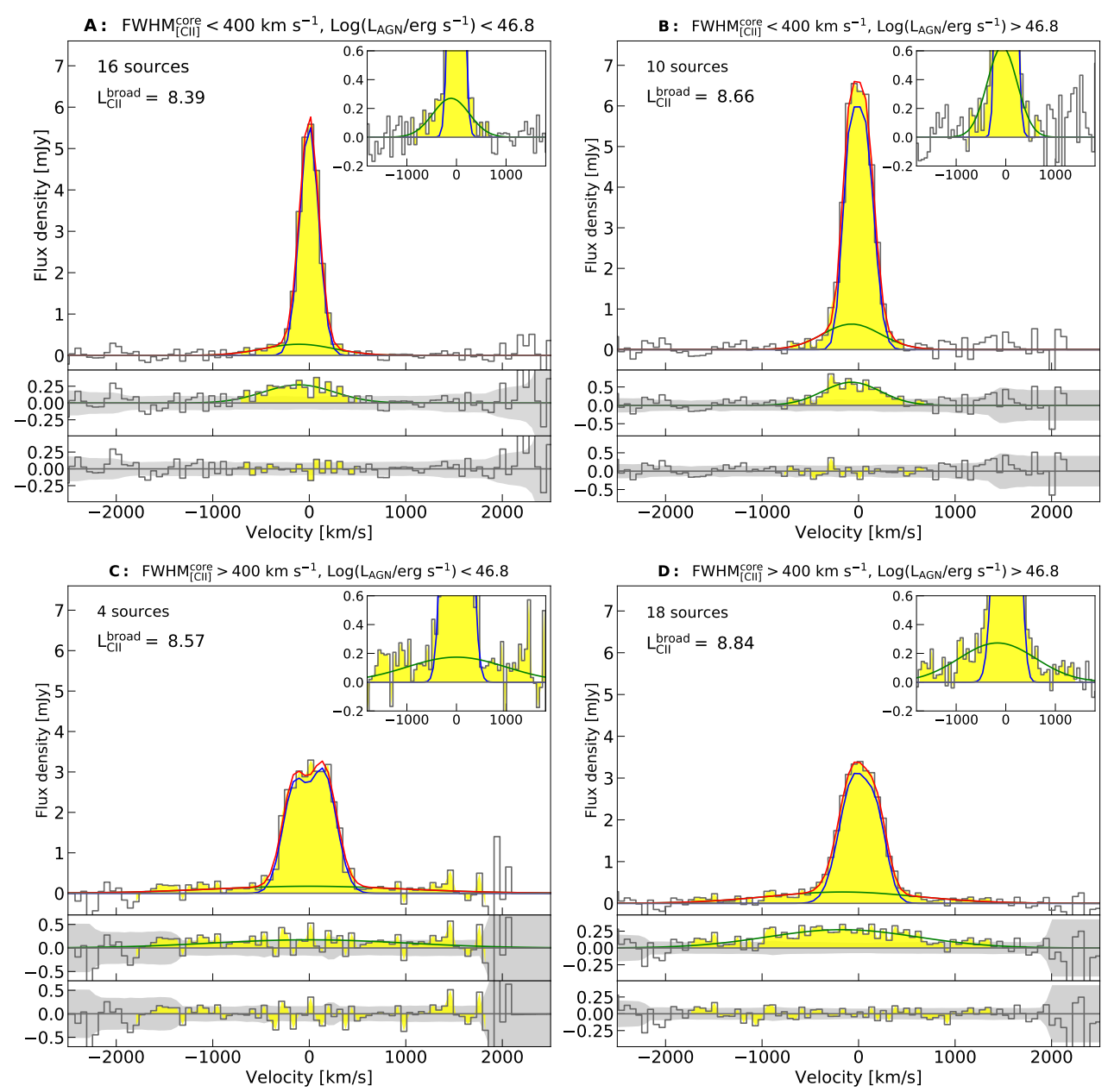

Fig. 4. Stacked integrated spectra for the different QSOs subgroups $A, B, C$, $D$ (properties of the individual samples are indicated in the top labels). For each plot, the first panel from top shows the [CII] flux density as a function of velocity, in bins of $60 \mathrm{~km} \mathrm{~s}^{-1}$. The red curve represents the best-fit 2 Gaussian components model; the two individual components are shown with blue and green curves. Labels indicate the number of stacked sources and the luminosity of the broad [CII] wings. The inset shows a zoom on the broad component. Second panel from top: residuals from the subtraction of the core component (blue line in first panel). The green curve shows the best fit broad component. Third panel: residuals from the two Gaussian components fitting. The $1 \sigma$ rms of the spectrum is also indicated by the shaded region.

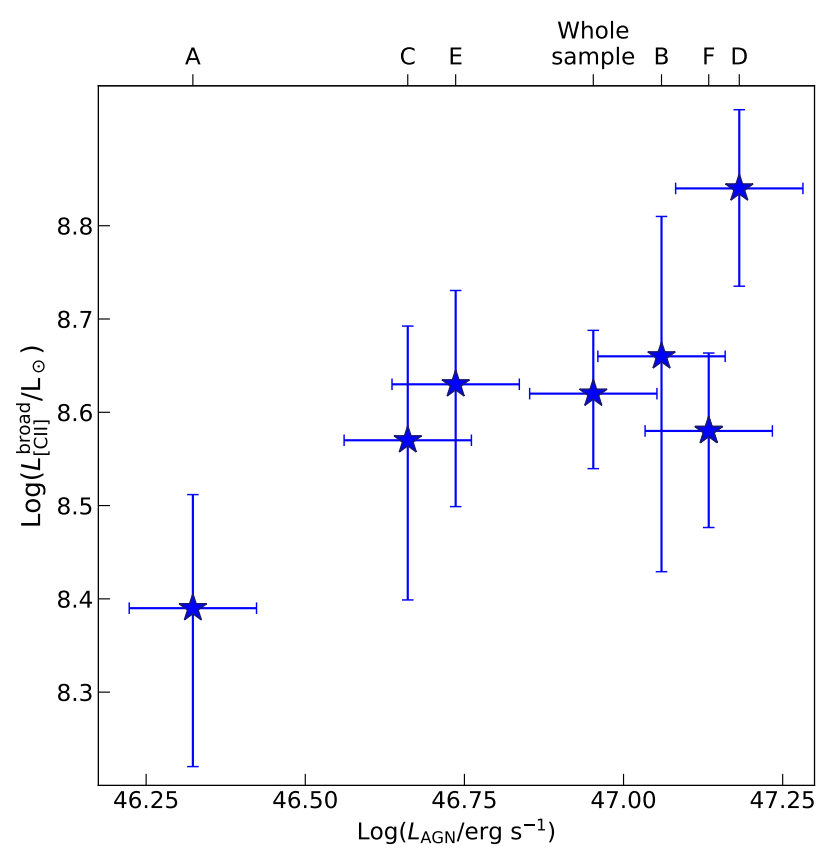

Fig. 5. Broad [CII] wings luminosity as a function of the AGN bolometric luminosity for the different stacks performed (indicated by the top labels). Error bars on $L_{\mathrm{AGN}}$ correspond to the 0.1 dex associated with the UV-based bolometric correction by Runnoe et al. (2012).

SFR is $\left.\sim 1750 M_{\odot} \mathrm{yr}^{-1}\right)$. However, the broad [CII] wings are well present in both stacks with comparable luminosity
$L_{[\mathrm{CII}]}^{\text {broad }} \sim 4 \times 10^{8} L_{\odot}$, indicating that SF does not significantly contribute to the outflows in the hosts of these powerful QSOs. Interestingly, as mentioned, we observe the largest blueshift $\left(\sim 240 \mathrm{~km} \mathrm{~s}^{-1}\right)$ of the broad [CII] wings in the high-SFR QSOs, which are those hosted in dustier galaxies, hence possibly corroborating the interpretation that the blueshift of the [CII] broad component is associated with heavy obscuration by the host galaxy. For stacks $E$ and $F$ we calculate an average signal-to-noise ratio of a spurious broad component of $\sim 0.4$ and $\sim 0.5$, respectively (see Sect. 3 ). Moreover, we estimate the contribution to $L_{[\mathrm{CII}]}^{\text {broad }}$ due to the combination of [CII] profiles with different FWHM to be only $\sim 10 \%$ for stack $E$ and $\sim 20 \%$ for stack $F$.

\subsection{Outflow detectability}

As mentioned in Sect. 1, up to date few tens of high- $z$ QSOs have been targeted in [CII], some of them with deep ALMA observations. Despite this fact, J1148+5251 remains the only source where a massive cold outflow was detected by Maiolino et al. (2012) and Cicone et al. (2015).

Among the deepest observations Venemans et al. (2017) targeted the [CII] emission in the $z=7.1$ QSO J1120+0641, with no detection of fast [CII] emission. The sensitivity reached by Venemans et al. (2017) is comparable to that of our B and $\mathrm{C}$ subgroups, where the broad [CII] wings are only marginally detected (see Sect. 4.2). A forthcoming work reaching similar depths (Carniani et al., in prep.) but exploiting configurations more sensitive to the extended, diffuse emission (hence more 

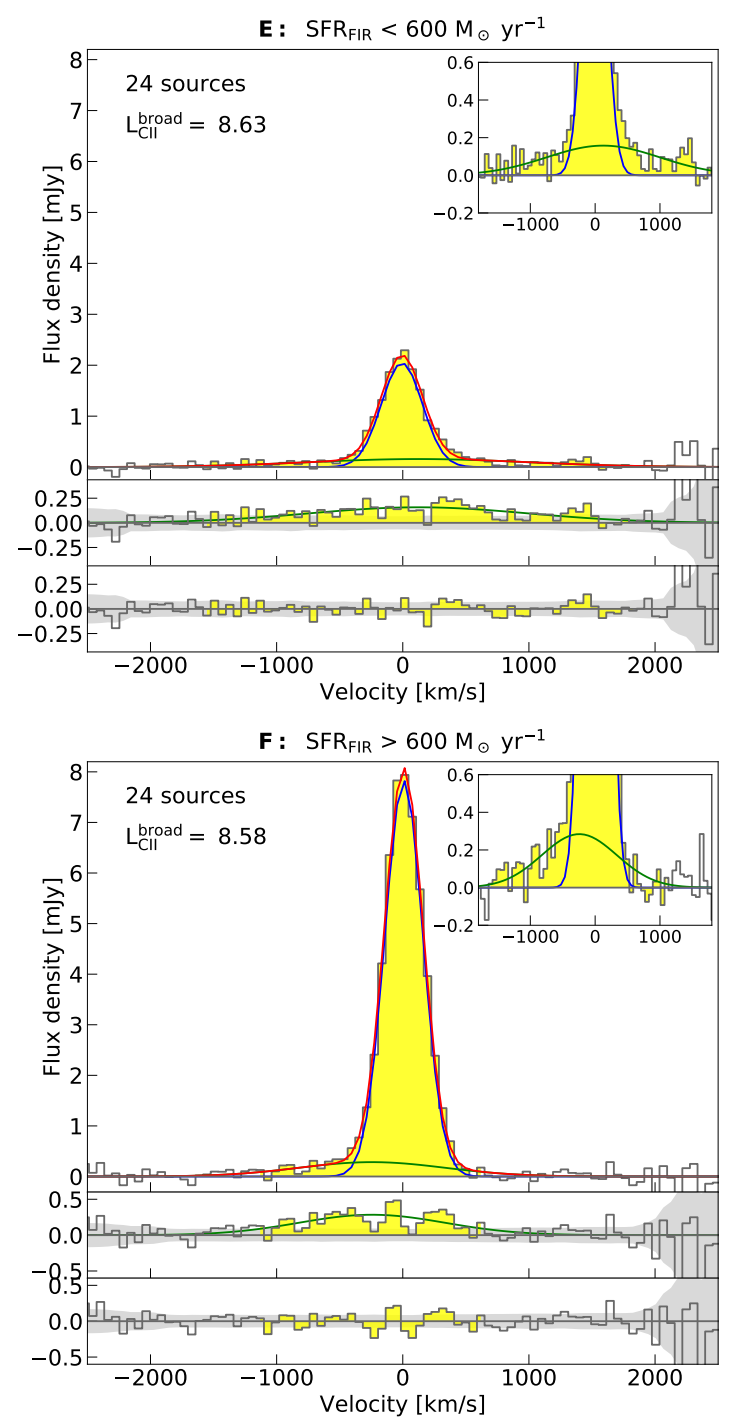

Fig. 6. Stacked integrated spectra for the low SFR $(E)$ and high SFR $(F)$ subgroups (properties of the individual samples are indicated in the top labels). For each plot, the first panel from top shows the [CII] flux density as a function of velocity, in bins of $60 \mathrm{~km} \mathrm{~s}^{-1}$, and the associated 2-Gaussians best-fit model; the two individual components are shown with blue and green curves. Labels indicate the number of stacked sources and the luminosity of the broad [CII] wings. The inset shows a zoom on the broad component. Second panel from top: residuals from the subtraction of the core component (blue line in first panel). The green curve shows the best fit broad component. Third panel: residuals from the two Gaussian components fitting. The $1 \sigma \mathrm{rms}$ of the spectrum is also indicated by the shaded region.

suitable to detect extended outflows), will present two QSOs were [CII] fast emission associated with AGN-driven outflows may be present. Similarly to our work, Decarli et al. (2018) computed the variance-weighted stacked spectrum of a sample of 23 ALMA [CII]-detected QSOs but finding no emission in excess of a Gaussian profile. However, the observations presented by Decarli et al. (2018) consist of very short ( $~ 8 \mathrm{~min})$ integrations and, therefore, sensitivities from $\sim 0.5$ to $1.0 \mathrm{mJy} \mathrm{beam}^{-1}$ covering the high-rms half of our sample. These observations correspond to $\sim 10 \%$ of the total on-source time covered by the QSOs in our sample. By applying our stacking procedure (Sect. 3) to the Decarli et al. (2018) sample alone, we derive a median rms sensitivity of 0.17 mJy beam $^{-1}$, comparable to that reached by subsamples $B$ and $C$, in which we find only marginal presence of [CII] wings. We note that our approach differs from that by Decarli et al. (2018) as they extract the individual QSOs spectra from the brightest pixel, while we choose extraction apertures of four ALMA beams recovering emission from extended scales. By fitting a two-Gaussian components model to the resulting stacked spectrum, we find a $S / N_{\text {[CII] }}^{\text {broad }} \sim 2$. Accordingly, we agree with Decarli et al. (2018) in finding no clear evidence of outflow signatures when stacking only their sample.

In our stacked spectra we limited the contamination from companions that are usually observed around a fraction of high- $z$, high-luminosity QSOs, that can be as high as $50 \%$ (e.g. Trakhtenbrot et al. 2017; Fan et al. 2018). Companions might in fact mimic a tail in the [CII] line profile similar to the broad [CII] wings indicative of outflowing gas. Most of these companions are located at much larger angular separations than the extraction regions of our spectra (see Sect. 4.1) and, therefore, do not contaminate our spectra. However, a few of the high- $z$ QSOs in our sample are known to have close companions with angular separation of about 1-2 arcsec which, despite the small extraction region used in our work, may partly contaminate the QSO emission. Specifically, the QSOs PJ231-20 and PJ308-21 from Decarli et al. (2017) show a close companion galaxy with a [CII] luminosity comparable to that of the QSO. In both cases the companion is slightly redshifted and may consequently contaminate the red wing of the [CII] stacked spectrum. The QSO PJ167-13 presented by Willott et al. (2017) is also likely associated with a companion at 0.9 arcsec separation, whose [CII] blueshifted $\left(\sim 270 \mathrm{~km} \mathrm{~s}^{-1}\right)$ emission corresponds to about $20 \%$ of the QSO [CII] luminosity. As mentioned in Sect. 3, individual sources do not significantly affect the luminosity of the whole sample stacked spectrum. As further verification, we also excluded these particular three QSOs from the stack and found no significant variation in the luminosity of the broad [CII] wings.

In our stacking procedure we assumed no relation between the luminosity of the broad [CII] wings and the AGN-host galaxy properties, such as the luminosity of the core of the [CII] emission line. Therefore, as mentioned in Sect. 3, we performed a standard variance weighted stack in flux density units. However, as our sample spans a factor of $\sim 1.7$ in luminosity distance, we verified that our results persist if performing a stack in luminosity density (see Appendix B). We derived a $L_{[\mathrm{CII}]}^{\text {broad }} \sim 4.7 \times 10^{8} L_{\odot}$, comparable to that found in the original stacked spectrum (Table 2).

\subsection{Stacked cube}

In this section we present the results from the stacking of the ALMA data cubes for the QSOs in our sample. We produced a stacked cube by applying the stacking technique presented in Sect. 3, that is we used Eqs. (1) and (2) to compute the varianceweighted stacked flux density of each spaxel in the final cube. It is a very simple approach primarily aimed at investigating the spatial scale of the [CII] outflows in the high-z QSOs host galaxies. Differently from the analysis of the integrated spectra of Sect. 4.1, here we did not not choose an extraction region but only piled up the emission contributions to each pixel of the map.

However, the application of this stacking method to heterogeneous observations may lead to a few issues, as discussed in the following. Firstly, combining observations with different angular resolutions (see Fig. 1) implies that emission from different physical scales may contribute to the total flux density of a same pixel. Degrading the observations to the lowest angular resolution would allow to stack emission arising from similar physical scales (given that the physical-to-angular scale ratio changes 

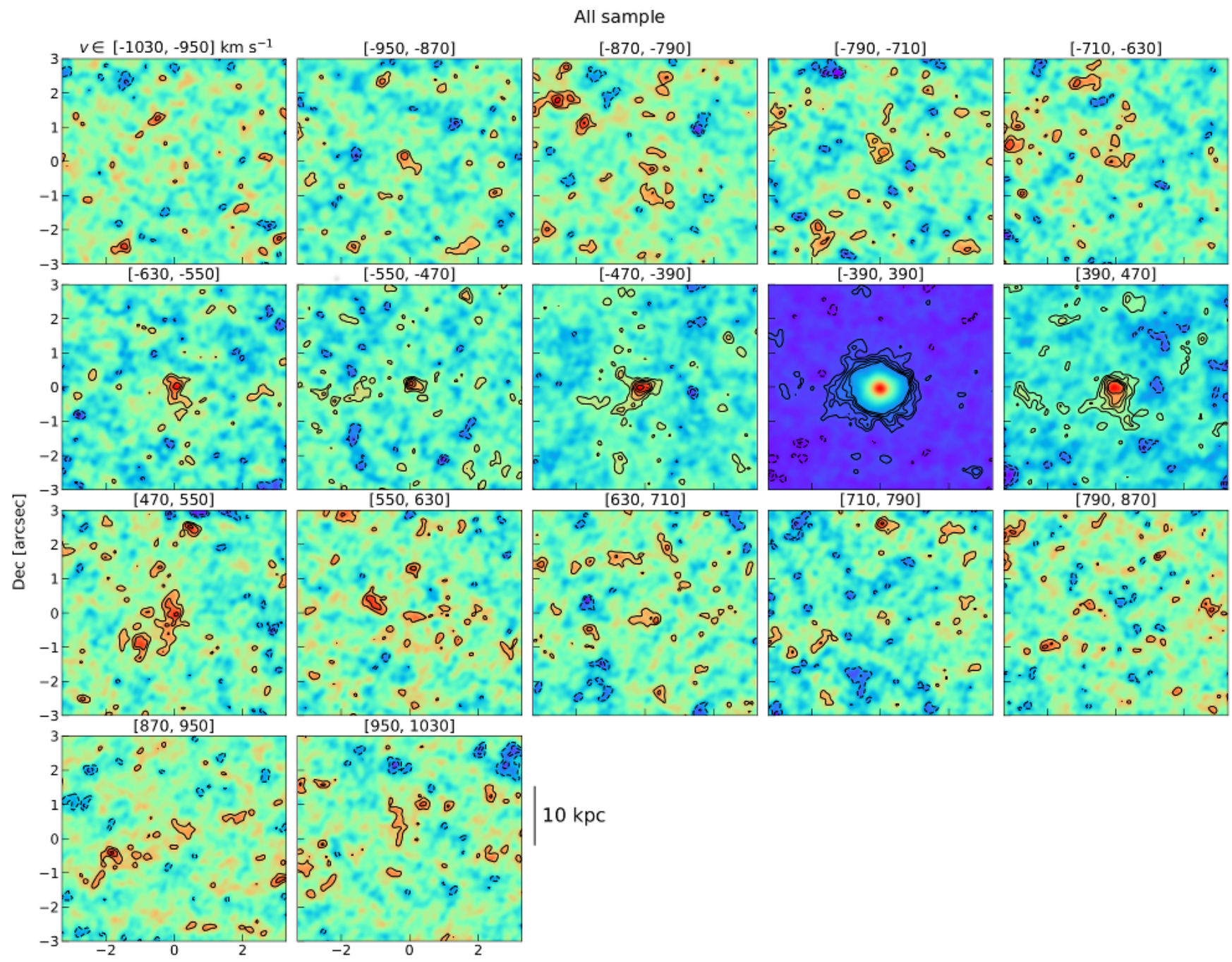

RA [arcsec]

Fig. 7. Channel maps of the whole sample stacked cube, corresponding to the central $6^{\prime \prime} \times 6^{\prime \prime}$ in the velocity range $v \in[-1000,1000] \mathrm{km} \mathrm{s} \mathrm{s}^{-1}$ (in bins of $80 \mathrm{~km} \mathrm{~s}^{-1}$, as indicated by the top labels). The bulk of the [CII] core emission is collapsed in the channel $v \in[-390,390] \mathrm{km} \mathrm{s}{ }^{-1}$. Contours correspond to $[-3,-2,2,3,4,5,6] \sigma$, where $\sigma$ is the rms sensitivity evaluated for each channel.

only by a factor $\lesssim 1.3$ in the redshift range of our sources). However, in interferometric data this would imply tapering the visibilities, that is lowering the weight of the extended baselines to the final map, at the expenses of the sensitivity. Therefore, we preferred not to modify the angular resolutions. However, by computing the variance-weighted beamsize of our stacked cube, it is possible to have an indication of the angular scale above which the most of the emission is resolved. For the allsample stacked cube we computed an average angular resolution $\theta_{\text {res }}=0.52^{\prime \prime} \times 0.68^{\prime \prime}$. In the case of a point source emission, the flux density contribution to the scale of the beam axes is only $\sim 6 \%$. We may therefore safely assess that emission on larger scales is mainly associated with extended, resolved emission.

Secondly, lacking a priori information about the structure and orientation of possible [CII] extended emission, in particular at high velocities, may cause outflowing anisotropic or clumpy [CII] emission to be diluted in the stack. As a consequence, the true fraction of $[\mathrm{CII}]$ emission associated with extended structures may be significantly higher.

The different angular resolutions of the interferometric observations are the result of different array configurations, which may filter out emission on different large angular scales.
As a rough estimate the largest angular scale (LAS) that can be recovered by interferometric observations is $L A S \sim(4-6) \times \theta_{\text {res }}$, where $\theta_{\text {res }}$ is the angular resolution. In the case of our stacked cube the flux loss of extended emission due to filtering starts to become important at $\sim 2$ arcsec.

Aware of these potential issues, Fig. 7 shows the central $6^{\prime \prime} \times 6^{\prime \prime}$ region of the stacked cube obtained by combining all the high- $z$ QSOs in our sample. Specifically, channel maps of the [CII] emission are shown for a velocity range $v \in[-1000$, $1000] \mathrm{km} \mathrm{s}^{-1}$ in bins of $80 \mathrm{~km} \mathrm{~s}^{-1}$. The bulk of the [CII] core emission is in the central $v \in[-390,390] \mathrm{km} \mathrm{s}^{-1}$. Compact [CII] emission is observed in almost all channels at $\gtrsim 2 \sigma$ up to $\sim 6 \sigma$, in addition to the presence of few offset clumps. At $|v| \sim 400-600 \mathrm{~km} \mathrm{~s}^{-1}$ there is also some indication of extended [CII] emission.

The channel maps of Fig. 7 suggest that we might be observing [CII] emission clumps moving at different velocities and characterised by a range of velocity dispersions. To build a global picture of the [CII] outflows, we created an integrated luminosity map of the high-velocity [CII] emission by summing the emission contributions, in the $80 \mathrm{~km} \mathrm{~s}^{-1}$ channel maps, detected at $>3 \sigma$ significance for at least three channels (i.e. 


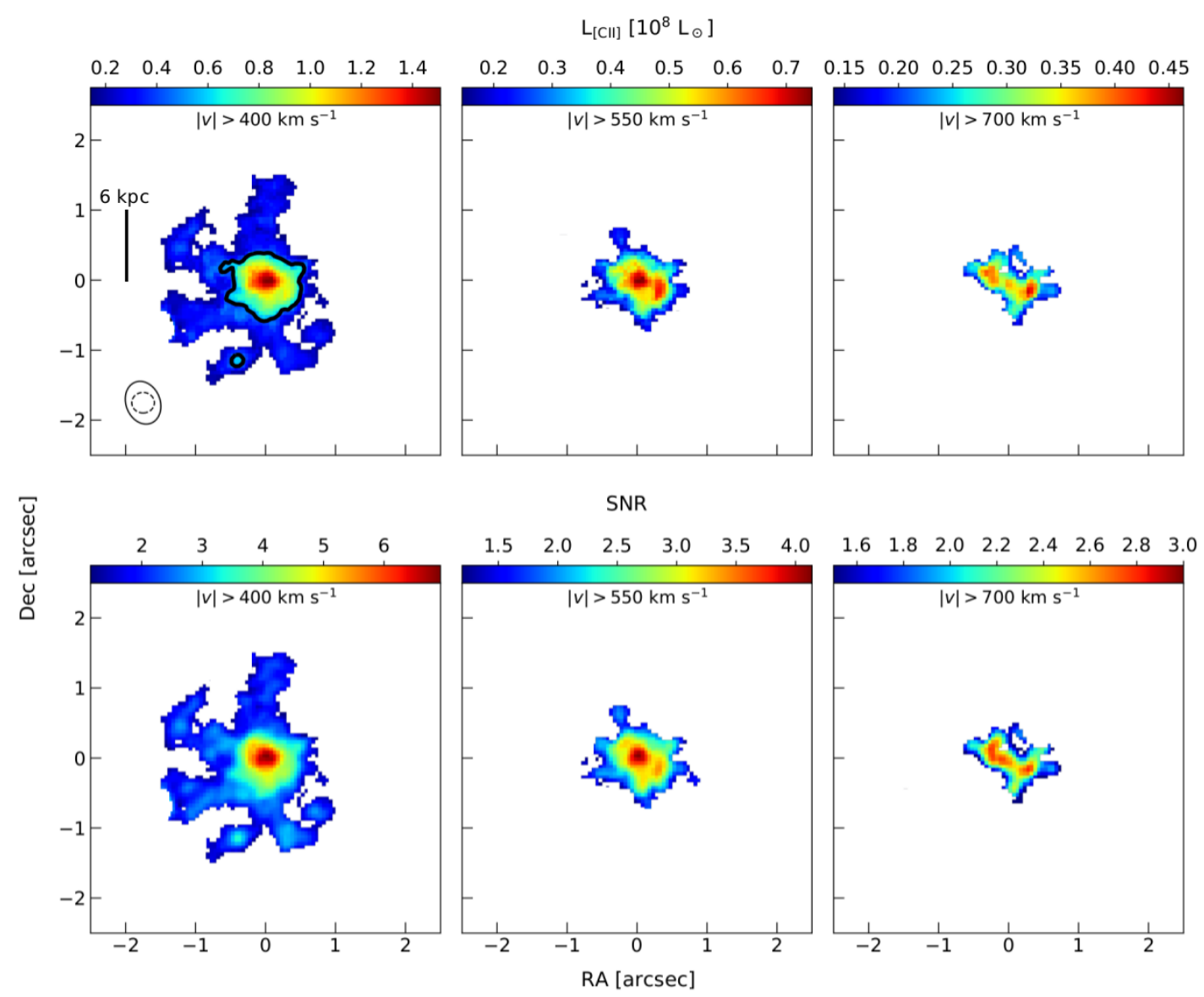

Fig. 8. Top: luminosity maps of the high-velocity [CII] emission derived from the whole sample stacked cube. From left to right panels: emission at increasing absolute velocities, specifically $|v|>400 \mathrm{~km} \mathrm{~s}^{-1},|v|>550 \mathrm{~km} \mathrm{~s}^{-1}$ and $|v|>700 \mathrm{~km} \mathrm{~s}^{-1}$. Maps were obtained by summing the emission at $>3 \sigma$ in $80 \mathrm{~km} \mathrm{~s}^{-1}$ channel maps for at least three channels (i.e. $\gtrsim 250 \mathrm{~km} \mathrm{~s}^{-1}$ ). The variance-weighted beam of the stacked cube is also indicated in the first map (solid line), together with the smallest beam contributing to the stack (dashed line). The thick solid contour encloses the region from which $50 \%$ of $L_{[\mathrm{CII}]}^{\text {broad }}$ arises. Bottom: signal-to-noise maps associated with the different velocity bins.

$\gtrsim 250 \mathrm{~km} \mathrm{~s}^{-1}$ ) in the whole-sample stacked cube. The result is shown in Fig. 8, where the maps corresponding to the velocity bins $|v|>400 \mathrm{~km} \mathrm{~s}^{-1},|v|>550 \mathrm{~km} \mathrm{~s}^{-1}$ and $|v|>700 \mathrm{~km} \mathrm{~s}^{-1}$ are displayed. We also plotted the associated signal-to-noise ratio maps.

As expected, most of the fast [CII] emission arises from the central regions, where all sources contribute in the stack. At the highest velocities $\left(>700 \mathrm{~km} \mathrm{~s}^{-1}\right)$ the nuclear outflow is still present at $\sim 3 \sigma$ significance. At moderate velocities $|v| \sim 400-550 \mathrm{~km} \mathrm{~s}^{-1}$, we observe extended emission up to $\sim 1.5$ arcsec, corresponding to $\sim 9 \mathrm{kpc}$ at $\left\langle z_{\text {stack }}\right\rangle=5.8$, and fully resolved compared with the average beam of the observations in the stack. We cannot exclude that part of this extended emission is due to contamination from the [CII] core emission. Marginally resolved emission is observed also at higher velocities. However, we stress that we might be losing a significant part of the extended emission in our stack. It is interesting that the apparent size of the outflow appears to decrease as a function of velocity. This is what is expected in an approximate spherically symmetric outflow as a consequence of projection effects. On the other hand, the stacked cube is a combination of outflows which may have different size, morphology and orientation between sources. The modest significance of the stacked data does not allow to draw conclusions on the outflow geometry.

Nonetheless, we can compute the spatial scale at which the bulk of the observed fast [CII] is emitted as the half light radius of the $|v|>400 \mathrm{~km} \mathrm{~s}^{-1}$ map (see Fig. 8), which has the high- est $\mathrm{S} / \mathrm{N}$. This radius corresponds to the average extent of the region enclosing $50 \%$ of the $[\mathrm{CII}]$ emission, indicated by the black contour in Fig. 8. We derived a beam-deconvolved half light radius $R_{\text {out }}=\sqrt{\left(R_{50 \%}^{2}-R_{\text {beam }}^{2}\right)} \sim 0.60 \operatorname{arcsec}$, where $R_{\text {beam }}$ is the weighted beam radius of the stacked cube. $R_{\text {out }}$ corresponds to $\sim 3.5 \mathrm{kpc}$ at $\left\langle z_{\text {stack }}\right\rangle$.

To ensure that the presence of spatially extended [CII] emission in the stacked cube was not an artefact of the combination of different ALMA beamsizes, we performed the whole sample stack of the data cubes after degrading the observations to a common angular resolution. We produced ALMA cubes at the worse angular resolution in our sample by tapering the visibilities to 1.2 arcsec. We did not consider observations with angular resolution $<0.6^{\prime \prime}$, as tapering to a worse resolution by a factor of $>2$ would result into a major loss of the original flux. The channel maps from the resulting stacked cube are shown in Fig. 9. The corresponding integrated luminosity map of the highvelocity emission is shown in Fig. 10. In agreement with Fig. 8, we found that the high-velocity [CII] emission is mainly located in a bright central component and extends up to $\sim 1.5-2$ arcsec. Indeed, combining the observations associated with the shorter baselines in our sample allowed us to recover some extended [CII] emission also in the high-velocity $\left(|v|>550 \mathrm{~km} \mathrm{~s}^{-1}\right.$ and $|v|>700 \mathrm{~km} \mathrm{~s}^{-1}$ ) bins. By following the same procedure presented in Sect. 4.4, we derived a beam-corrected half light radius $R_{\text {out }}^{\text {taper }} \sim 4.6 \mathrm{kpc}$ at the representative redshift of the stack 


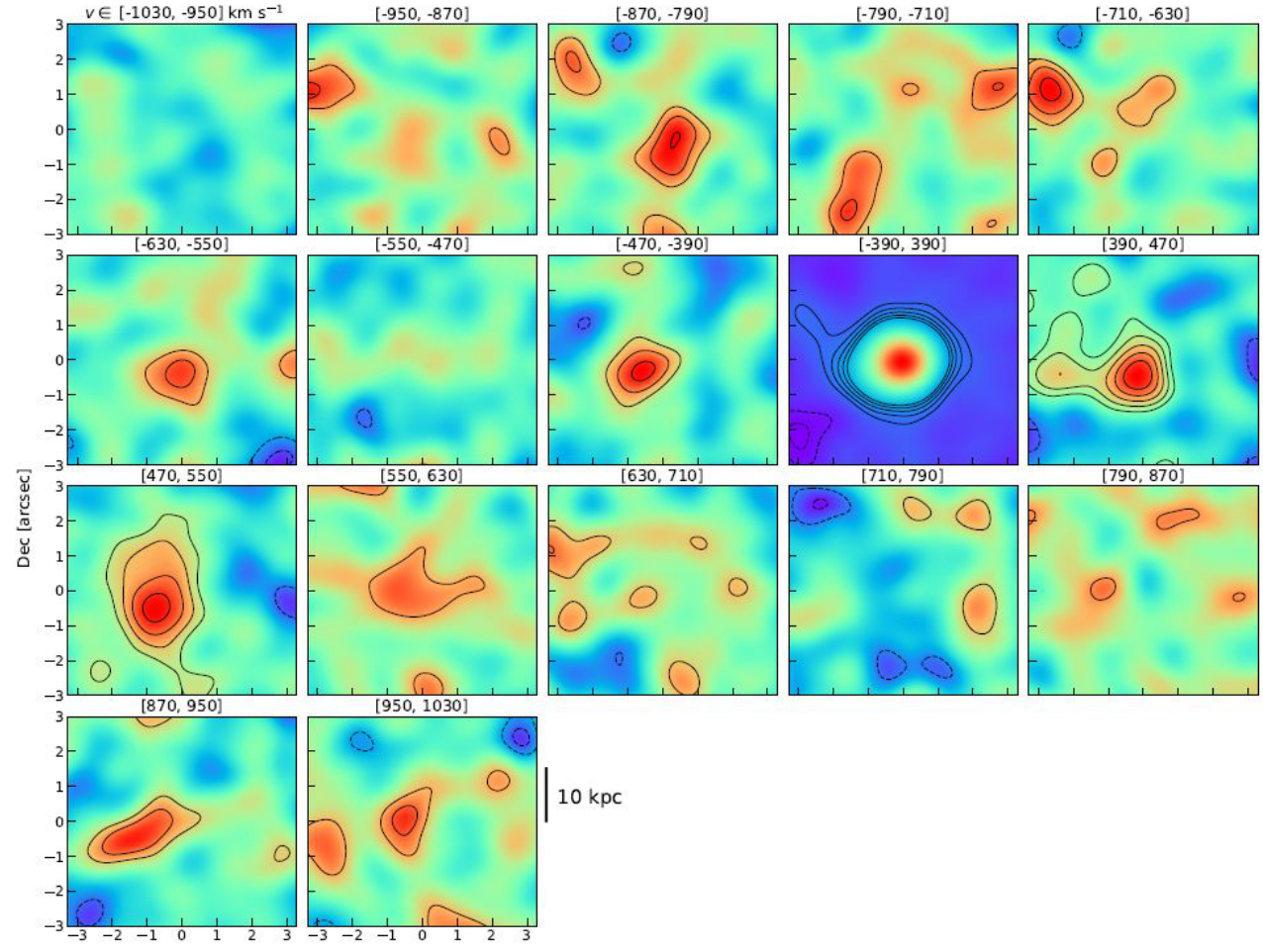

RA [arcsec]

$\mathrm{L}_{\text {[CII] }}\left[10^{8} \mathrm{~L}_{\odot}\right]$
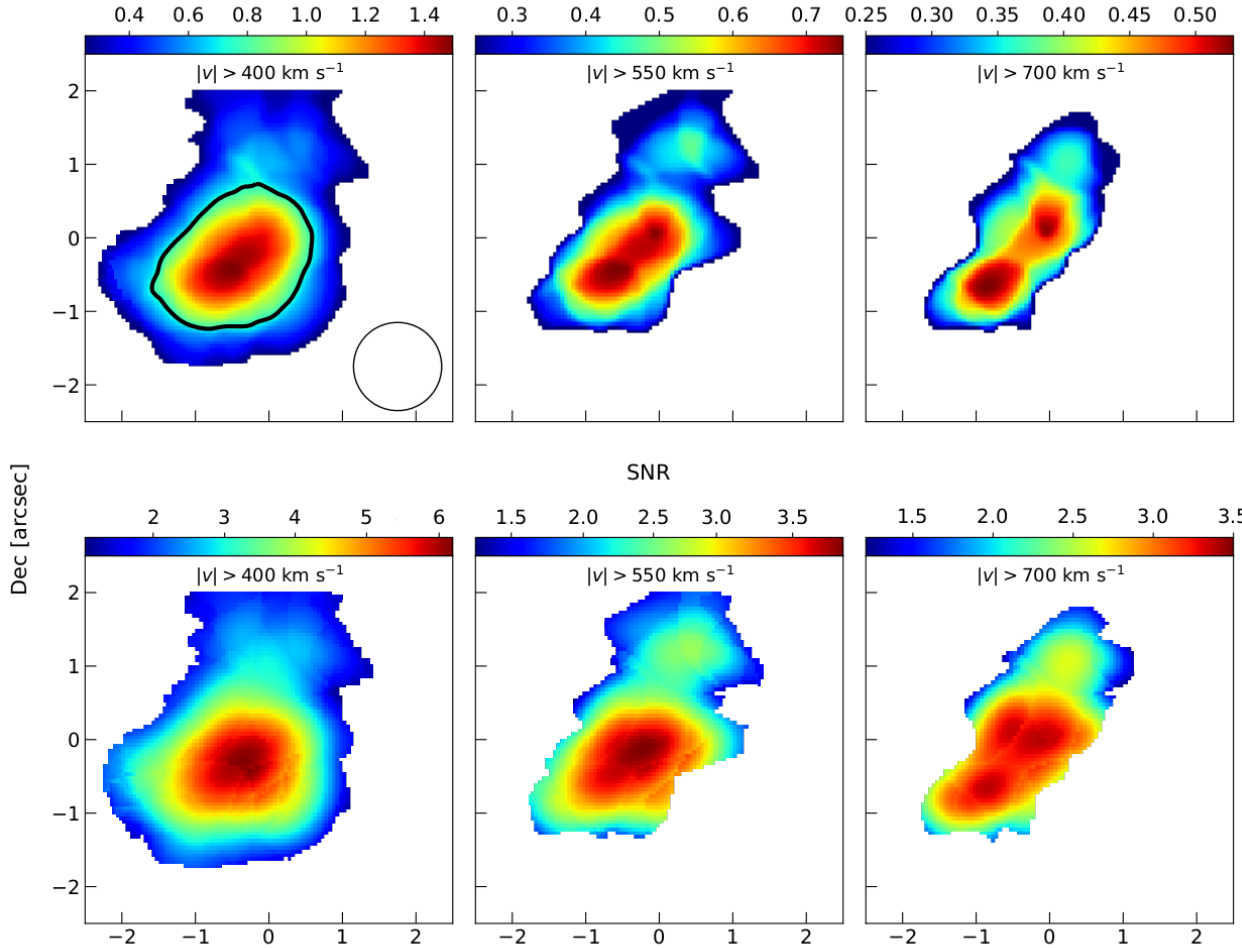

SNR

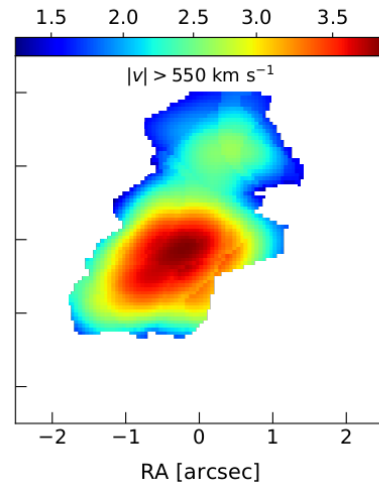

1.5

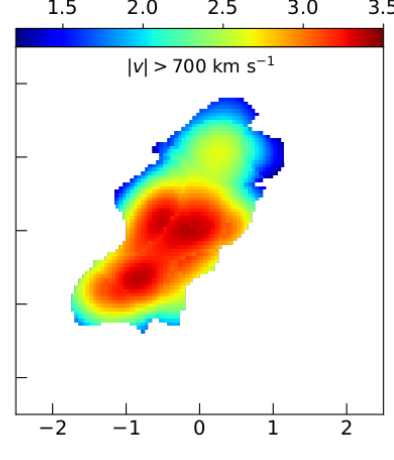

Fig. 9. Channel maps of the stacked cube obtained by removing the higher resolution $(<0.6$ arsec) observations (see Sect. 2) and tapering the remaining data to a common resolution of 1.2 arcsec. The displayed region corresponds to the central $6^{\prime \prime} \times 6^{\prime \prime}$ in the velocity range $v \in[-1000,1000] \mathrm{km} \mathrm{s}^{-1}$ (in bins of $80 \mathrm{~km} \mathrm{~s}^{-1}$, as indicated by the top labels). The bulk of the [CII] core emission is collapsed in the channel $v \in[-390,390] \mathrm{km} \mathrm{s}^{-1}$. Contours correspond to $[-3,-2,2,3,4,5,6] \sigma$, where $\sigma$ is the rms sensitivity evaluated for each channel.

Fig. 10. Top: luminosity maps of the high-velocity [CII] emission derived from the stacked cube of the $\gtrsim 0.6$ arcsec sample, after having applied a tapering to a common 1.2 arcsec resolution. From left to right panels: emission at increasing absolute velocities, specifically $|v|>$ $400 \mathrm{~km} \mathrm{~s}^{-1},|v|>550 \mathrm{~km} \mathrm{~s}^{-1}$ and $|v|>$ $700 \mathrm{~km} \mathrm{~s}^{-1}$. Maps were obtained by summing the emission at $>3 \sigma$ in $80 \mathrm{~km} \mathrm{~s}^{-1}$ channel maps for at least three channels (i.e. $\gtrsim 250 \mathrm{~km} \mathrm{~s}^{-1}$ ). The 1.2 arcsec beam is also indicated in the first map (solid line). The thick solid contour encloses the region from which $50 \%$ of $L_{[\mathrm{CII}]}^{\text {broad }}$ arises. Bottom: signal-to-noise maps associated with the different velocity bins. $\left\langle z_{\text {stack }}^{\text {taper }}\right\rangle \simeq 6.2$, slightly larger than the radius inferred from the stack of the total sample.

\section{Discussion}

As mentioned in Sect. 1, most of [CII] emission in IR-bright galaxies is expected to arise from PDRs (e.g. Sargsyan et al.
2012), accounting for about $70 \%$ of the total [CII] emission. Under the assumption that [CII] emission is optically thin, it is possible to link the luminosity of the broad [CII] wings to the mass of the outflowing atomic gas. In case of optically thick [CII], the true outflowing gas mass would be larger. It is therefore possible to estimate the typical energetics of [CII] outflows in high-redshift, high-luminosity QSOs, in the central $\sim 3 \mathrm{kpc}$ 
Table 3. Outflow parameters associated with the different stacked integrated spectra.

\begin{tabular}{|c|c|c|c|c|c|c|}
\hline $\begin{array}{l}\text { Stack } \\
(1)\end{array}$ & & $\begin{array}{c}v_{\text {out }} \\
{\left[\mathrm{km} \mathrm{s}^{-1}\right]} \\
(2)\end{array}$ & $\begin{array}{c}M_{\text {out }} \\
{\left[10^{8} M_{\odot}\right]} \\
(3)\end{array}$ & $\begin{array}{c}\dot{M}_{\text {out }} \\
{\left[M_{\odot} \mathrm{yr}^{-1}\right]} \\
(4)\end{array}$ & $\begin{array}{c}\dot{E}_{\text {out }} \\
{\left[10^{43} \mathrm{erg} \mathrm{s}^{-1}\right]} \\
(5)\end{array}$ & $\begin{array}{c}\dot{P}_{\text {out }} / P_{\text {AGN }} \\
(6) \\
\end{array}$ \\
\hline Whole sample & & $960 \pm 120$ & $3.7 \pm 0.7$ & $100 \pm 20$ & $2.6 \pm 0.7$ & $0.20 \pm 0.05$ \\
\hline A & $F W H M_{[\mathrm{CII}]}<400 \mathrm{~km} \mathrm{~s}^{-1}, L_{\mathrm{AGN}}<10^{46.8} \mathrm{erg} \mathrm{s}^{-1}$ & $550 \pm 110$ & $2.4 \pm 0.9$ & $35 \pm 15$ & $0.30 \pm 0.15$ & $0.17 \pm 0.07$ \\
\hline $\mathrm{B}$ & $F W H M_{[\mathrm{CII}]}<400 \mathrm{~km} \mathrm{~s}^{-1}, L_{\mathrm{AGN}}>10^{46.8} \mathrm{erg} \mathrm{s}^{-1}$ & $440 \pm 90$ & $4.6 \pm 1.5$ & $55 \pm 20$ & $0.33 \pm 0.15$ & $0.04 \pm 0.02$ \\
\hline $\mathrm{C}$ & $F W H M_{[\mathrm{CII}]}>400 \mathrm{~km} \mathrm{~s}^{-1}, L_{\mathrm{AGN}}<10^{46.8} \mathrm{erg} \mathrm{s}^{-1}$ & $1180 \pm 380$ & $3.2 \pm 1.0$ & $115 \pm 50$ & $5.0 \pm 2.3$ & $0.58 \pm 0.24$ \\
\hline $\mathrm{D}$ & $F W H M_{[\mathrm{CII}]}>400 \mathrm{~km} \mathrm{~s}^{-1}, L_{\mathrm{AGN}}>10^{46.8} \mathrm{erg} \mathrm{s}^{-1}$ & $1100 \pm 140$ & $6.2 \pm 1.2$ & $185 \pm 35$ & $7.4 \pm 2.0$ & $0.28 \pm 0.07$ \\
\hline $\mathrm{E}$ & $\mathrm{SFR}_{\mathrm{FIR}}<600 M_{\odot} \mathrm{yr}^{-1}$ & $1210 \pm 230$ & $3.9 \pm 1.1$ & $135 \pm 40$ & $3.0 \pm 0.7$ & $0.50 \pm 0.12$ \\
\hline $\mathrm{F}$ & $\mathrm{SFR}_{\mathrm{FIR}}>600 M_{\odot} \mathrm{yr}^{-1}$ & $930 \pm 140$ & $3.6 \pm 0.8$ & $95 \pm 30$ & $2.5 \pm 0.8$ & $0.12 \pm 0.03$ \\
\hline
\end{tabular}

Notes. Columns give the following information: (1) stacked sample, (2) outflow velocity (3) atomic gas mass associated with the broad [CII] wings, (4) mass outflow rate, computed following Fluetsch et al. (2019), (5) kinetic power and (6) momentum load factor of the outflow.

regions (see Sect. 4.4). Specifically, to compute the outflow mass of atomic neutral gas we use the relation from Hailey-Dunsheath et al. (2010):

$M_{\text {out }} / M_{\odot}=0.77\left(\frac{0.7 L_{[\mathrm{CII}]}}{L_{\odot}}\right)\left(\frac{1.4 \times 10^{-4}}{X_{\mathrm{C}^{+}}}\right) \times \frac{1+2 e^{-91 K / T}+n_{\text {crit }} / n}{2 e^{-91 K / T}}$

where $X_{\mathrm{C}^{+}}$is the $\mathrm{C}^{+}$fraction per hydrogen atom, $T$ is the gas temperature, $n$ is the gas density and $n_{\text {crit }} \sim 3 \times 10^{3} \mathrm{~cm}^{-3}$ is the [CII] $\lambda 158 \mu \mathrm{m}$ critical density. We use Eq. (3) in the approximation of $n \gg n_{\text {crit }}$, thus deriving a lower limit on the outflowing gas mass. This choice is in agreement with Maiolino et al. (2005) who estimated a gas density of $\sim 10^{5} \mathrm{~cm}^{-3}$ in $\mathrm{J} 1148+5251$, but also confirmed by the large densities typically observed in QSO outflows (Aalto et al. 2012, 2015), and allows us to directly compare with the energetics of the outflow detected in this QSO. Following Maiolino et al. (2012) and Cicone et al. (2015) we consider a conservative $X_{\mathrm{C}^{+}} \sim 10^{-4}$ and a gas temperature of $200 \mathrm{~K}$, both typical of PDRs (HaileyDunsheath et al. 2010). We recall that, although the molecular gas phase in the ISM has typically lower temperatures, in the outflow even the molecular gas is expected to have higher temperatures, of a few $100 \mathrm{~K}$ (Richings \& Faucher-Giguère 2018). Assuming a temperature from $100 \mathrm{~K}$ to $1000 \mathrm{~K}$ would imply a variation of only $20 \%$ in the resulting gas mass. The 0.7 factor in the first parenthesis of Eq. (3) accounts for the fraction of neutral [CII] typically arising from PDRs, while $30 \%$ typically comes from the partially ionised phase (Stacey et al. 2010; Maiolino et al. 2012; Cicone et al. 2014). By applying Eq. (3) to the stack of the whole sample we infer a mass of the outflowing neutral gas $M_{\text {out }}=(3.7 \pm 0.7) \times 10^{8} M_{\odot}$, see Table 3 .

To compute the [CII] outflows energetics of the high- $z$ QSOs in our sample, we assume the sceario of time-averaged expelled shells or clumps (Rupke et al. 2005):

$\dot{M}_{\text {out }}=\frac{v_{\text {out }} \times M_{\text {out }}}{R_{\text {out }}}$

where $v_{\text {out }}=\left|\Delta v_{\text {broad }}\right|+F W H M_{[\text {CII] }}^{\text {broad }} / 2$ (see Table 3) and $\Delta v_{\text {broad }}$ is the velocity shift of the centroid of the broad [CII] wings with respect to the systemic emission, $R_{\text {out }} \sim 3.5 \mathrm{kpc}$ as derived in Sect. 4.4 from the extension of the [CII] broad wings as inferred from the stacked cube. We calculate the kinetic power associated with the [CII] outflows as:

$\dot{E}_{\text {out }}=\frac{1}{2} \dot{M}_{\text {out }} \times v_{\text {out }}^{2}$ and the momentum load:

$\dot{P}_{\text {out }} / \dot{P}_{\mathrm{AGN}}=\frac{\dot{M}_{\text {out }} \times v_{\text {out }}}{L_{\mathrm{AGN}} / c}$

where $\dot{P}_{\mathrm{AGN}}=L_{\mathrm{AGN}} / c$ is the AGN radiation momentum rate. This approach allows us to directly compare our findings to the collection of 30 low redshift AGN by Fluetsch et al. (2019), for which the energetics of spatially resolved molecular and (in $\sim$ one third of the same sources) neutral [CII] and ionised outflows has been homogeneously calculated.

The resulting outflow parameters for the whole sample stack and the different subsamples considered (see Sect. 4.2) are listed in Table 3 . We derive a mass outflow rate of $\dot{M}_{\text {out }}=100 \pm 20 M_{\odot} \mathrm{yr}^{-1}$ for the stack of the whole sample, while for the large FWHM, high- $L_{\mathrm{AGN}}$ subgroup (stack $D$ ) we find $\dot{M}_{\text {out }} \sim 200 M_{\odot} \mathrm{yr}^{-1}$. These outflow rates only refer to the atomic neutral component. Fluetsch et al. (2019) obtained that, for AGN-driven outflows, the molecular mass outflow rates are of the same order as the atomic neutral outflow rates, while the contribution from the ionised gas is negligible, at least in the luminosity range probed by them. They find that the molecular-toionised outflow rate increases with luminosity, in contrast with what found by Fiore et al. (2017); the discrepancy may originate from the fact that the latter study investigate disjoint samples, or may originate from the different luminosity ranges sampled. If we assume that the relations found by Fluetsch et al. (2019) also apply to these distant luminous QSOs, then the implied total outflow rate is twice the value inferred from [CII].

Figure 11a shows the mass outflow rate as a function of the AGN bolometric luminosity. Stars show the atomic neutral outflow rate inferred from the [CII] broad wings for the various stacked spectra, as indicated in the legend. The circles, connected to the star through a dashed line, indicate the inferred outflow rate by accounting also for the molecular gas content in the outflow assuming the relation given by Fluetsch et al. (2019). Blue, green and purple squares show the molecular, ionised and atomic neutral outflow rates measured by Fluetsch et al. (2019) in local AGN. In the latter case the neutral component is obtained through [CII] observations of local galaxies performed by the Herschel infrared space telescope (Janssen et al. 2016) and purple circles show the effect of correcting the atomic outflow rate as discussed above. Hollow green squares show the ionised outflow rates inferred from Fiore et al. (2017); these are from a disjoint sample (they do not have measurements for the molecular and atomic phase) and may be subject to different selection effects, but they have the advantage to extend to much higher 

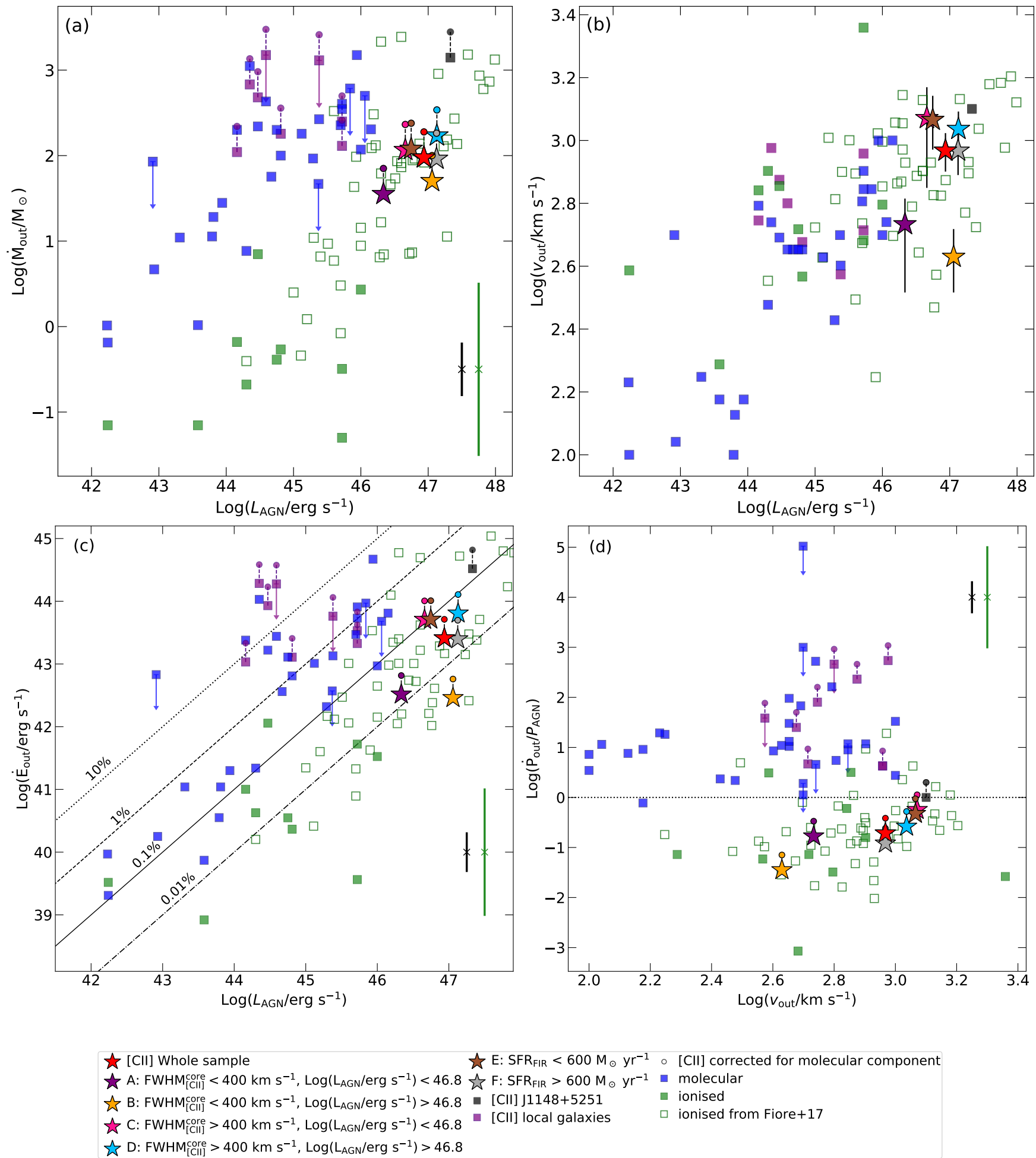

Fig. 11. [CII] outflows parameters. (a): Mass outflow rate as a function of $L_{\mathrm{AGN}}$ for the different stacked spectra (stars, see legend for details), compared to the sample of 30 low-redshift AGN from Fluetsch et al. (2019) for which spatially resolved molecular (blue) and, in one third of the sample, ionised (green) outflows have been observed. We also included the compilation of ionised outflows (hollow green squares) with spatial information in $z \sim 0.1-3$ AGN from Fiore et al. (2017), recomputed according to Eqs. (4)-(6). Purple squares are local systems for which the outflow has been traced in [CII] through observations with the Herschel Space Observatory (Janssen et al. 2016; Fluetsch et al. 2019). By applying the atomic-to-molecular outflowing gas mass correction by Fluetsch et al. (2019), the molecular+atomic mass outflow rates are shown with circles. The typical $\sim 0.3$ dex uncertainty on $\dot{M}_{\text {out }}$ for the [CII] outflows found in our $z \sim 6$ QSOs (similar to that of outflows in the atomic neutral and molecular phase in low- $z$ AGN) is shown by the black solid line, while the uncertainty on $\dot{M}_{\text {out }}$ for the ionised outflows is shown by the green line. (b): Outflow velocity as a function of $L_{\mathrm{AGN}}$. $(c)$ : Kinetic power as a function of $L_{\mathrm{AGN}}$. The dotted, dashed, solid and dot-dashed curves indicate kinetic powers that are $10 \%, 1 \%, 0.1 \%$ and $0.01 \%$ of the AGN luminosity. $(d)$ : Momentum load factor as a function of the outflow velocity. The horizontal line corresponds to $\dot{P}_{\mathrm{out}}=P_{\mathrm{AGN}}$. 
luminosities than the sample in Fluetsch et al. (2019). Figure 11a illustrates the well known phenomenon that the outflow rate increases with the AGN luminosity and that generally the outflow rate is dominated by the neutral phases (atomic and neutral). However, at the very high luminosities probed by our stacked spectra of the most distant QSOs the outflow rates associated with the neutral phase appear to deviate from the trend observed locally, and the outflow rates seem similar to those observed in the ionised phase. For completeness, Fig. 11b shows the outflow velocity as a function of the AGN luminosity, illustrating that the velocity of the outflow observed in the stacked spectra is consistent with the trend observed in other AGN and in other phases, further confirming that these outflows are QSO-driven.

Figure 11c shows the kinetic power as a function of the AGN luminosity with the same symbols as in Fig. 11a. For our stacked spectra, the kinetic power is between $0.01 \%$ and $0.5 \%$ of $L_{\mathrm{AGN}}$, that is much lower than what expected from AGN "energy-driven" outflow models ( $\dot{E}_{\text {out }} \sim 0.05 \times L_{\mathrm{AGN}}$, e.g. Di Matteo et al. 2005 ; Zubovas \& King 2012) which ascribe outflows to the nuclear winds that expands in an energy-conserving way.

Figure 11d shows the outflow momentum load factor, that is the outflow momentum rate relative to $\dot{P}_{\text {AGN }}$, as a function of the outflow velocity. For our stacked spectra $\dot{P}_{\text {out }} / \dot{P}_{\text {AGN }} \lesssim 1$, while energy-driven outflow models would expect momentum load factors of $\sim 20$. These results suggest that the outflows in these powerful quasars are either energy-driven but with poor coupling with the ISM of the host galaxy, or are driven by direct radiation pressure onto the dusty clouds (e.g. Ishibashi et al. 2018). In either cases the outflow unlikely is in the so-called ejective mode, i.e. very effective in removing gas from the entire galaxy, hence in completely suppressing star formation (Costa et al. 2015, 2018; Bourne et al. 2014, 2015; Roos et al. 2015; Gabor \& Bournaud 2014), although such ejective mode can be effective in clearing of the gas content and quenching star formation in the central regions. Moreover, the outflow can be effective in heating the circumgalactic medium and therefore preventing further accretion of fresh gas onto the galaxy, hence resulting in a delayed quenching of the galaxy by "starvation" (Peng et al. 2015).

It could also be, in contrast with what observed in the lowluminosity local AGN, that in these very luminous, distant QSOs the bulk of the outflow is highly ionised. The observation that in other very luminous QSOs the ionised outflow rate, kinetic power and momentum rate is similar to the same quantities locally observed in the molecular phase (Fig. 11), does suggest that the balance between the various phases is different in these systems (Bischetti et al. 2017; Fiore et al. 2017). However, as illustrated in Fig. 11, even the ionised phase does not seem to be massive and powerful enough to match the requirements of the energy-driven scenario with high coupling.

In alternative, the interferometric data used in our stack of the [CII] emission may miss extended, diffuse emission associated with outflows. Indeed, a large fraction of the data have angular resolution higher than $0.7^{\prime \prime}$, which may prevent them to detect emission on scales larger than $\sim 3-4^{\prime \prime}$. The lack of sensitivity to extended, diffuse emission may indeed be a major problem in very distant systems, due to the rapid cosmological dimming of the surface brightness, decreasing as $\sim(1+z)^{4}$. This scenario may also explain why the $[\mathrm{CII}]$ outflow rate and kinetic power in the stacked data of distant QSOs do not seem to increase significantly with respect to the local, lower-luminosity AGN (purple square symbols in Fig. 11) whose [CII] broad wings were observed with Herschel.

Within this context it is interesting to note that in the QSO $\mathrm{J} 1148+5251$ at $z=6.4$ Maiolino et al. (2012) and Cicone et al.
(2015) did detect a very extended outflow on scales of $\sim 6^{\prime \prime}$, by exploiting low angular resolution observations. J1148+5251 (black square in Fig. 11) is indeed characterised by a larger outflow rate and higher kinetic power with respect to the stacked measurements. However, even for J1148+5251 the kinetic power and momentum rate appear to be significantly lower than what expected by the simple scenario of energy-driven outflows with high coupling with the ISM.

\section{Conclusions}

In this work we have presented the stacking analysis of a sample of 48 QSOs at $4.5<z<7.1$ detected in [CII] by ALMA, equivalent to an observation of $\sim 34 \mathrm{~h}$ on-source, aimed at investigating the presence and the properties of broad [CII] wings tracing cold outflows. The stack allows us to reach an improvement in sensitivity by a factor of $\sim 14$ with respect to the previous observation of a massive [CII] outflow in J1148+5251 at $z \sim 6.4$ (Maiolino et al. 2012; Cicone et al. 2015).

From the stacked integrated spectra, we clearly detect broad [CII] wings, tracing cold outflows associated with $z \sim 6$ QSOs and whose velocities exceed $1000 \mathrm{~km} \mathrm{~s}^{-1}$. This weak, broad component has not been previously detected in single observations (except for the case of J1148+5251) because of insufficient sensitivity. The same limitation applies to the stack recently performed by Decarli et al. (2018) on the sample of $23 z \sim 6$ QSOs with ALMA [CII] detection, which were mostly observed with very short (few minutes) exposures. In fact similarly to Decarli et al. (2018), we find no significant broad [CII] wings in the stacked spectrum of their sources alone.

The redshifted [CII] wing is fainter than the blueshifted [CII] wing. This may be associated with the asymmetric distribution of the spectral coverage of the spectra used in the stacked spectrum. However, if confirmed with additional data, this asymmetry would suggest that in these systems the dusty gas in the host galaxy has a column density high enough to obscure the receding component of the outflows, with respect to our line of sight. High dust column densities capable of absorbing even at far-IR and sub-mm wavelengths have been observed in local ULIRGs.

By splitting the sample in AGN luminosity and SFR bins, we observe that the strength of the stacked broad component correlates with the AGN luminosity, but does not depend on the SFR. This indicates that the QSOs are the primary driving mechanism of the [CII] outflows in these systems. Moreover, we find that the broad component is very blushifted in the stack with high SFR and nearly symmetric in the stack with low SFR. Since the SFR correlates with the gas and dust content in the galaxy, this finding corroborates the interpretation that the blueshift of the [CII] broad component might be associated with heavy dust absorption.

By stacking the ALMA data cubes, we investigate the morphology of the [CII] outflows in our sample and find that the high-velocity [CII] emission extends up to $R_{\text {out }} \sim 3.5 \mathrm{kpc}$. However, we cannot exclude that additional, more extended emission is present but missed by the interferometric data used for the stacking. Moreover, averaging outflows with different orientations and clumpiness may result into dilution effects affecting the observed intensity and extension of the [CII] broad wings in the stacked cube.

From the stacked cube we infer an average atomic mass outflow rate $\dot{M}_{\text {out }} \sim 100 M_{\odot} \mathrm{yr}^{-1}$, which doubles for the stack of the most luminous sources. By correcting for the atomicto-molecular gas ratio found by Fluetsch et al. (2019), the former value translates into a total mass outflow rate of about 
$200 M_{\odot} \mathrm{yr}^{-1}$. The associated kinetic powers are consistent with $0.1 \%$ of $L_{\mathrm{AGN}}$ for most stacks, while momentum load factors span the range $0.1-1$; these $\dot{M}_{\text {out }}$ are lower than what observed in cold outflows associated with local, lower luminosity AGN, and are lower than the expectations of standard energy-driven outflow models (hence indicating either a low coupling with the ISM and/or a different driving mechanism, such as direct radiation pressure on the dusty clouds).

As a consequence, QSO-driven outflows in the early universe may have not been very effective in clearing the galaxy from their gas content, although they may have been effective in clearing and quenching their central regions, and also heating the galaxy halo hence resulting into a delayed star formation quenching as a consequence of starvation.

Future deep ALMA follow-up observations will allow us to confirm the presence of [CII] outflows in individual high- $z$ QSOs. Furthermore, the increasing number of available sources on the ALMA archive will increase the statistics, enabling us to reduce the uncertainties on the cold outflows parameters in the early Universe.

Acknowledgements. We are grateful to the anonymous referee for valuable feedback that helped us to improve the paper. This paper makes use of the following ALMA data: ADS/JAO.ALMA\#2011.0.00243.S, 2012.1.00604.S 2012.1.00676.S, 2012.1.00882.S, 2013.1.01153.S, 2015.1.01115.S and 2016.1.01515.S. ALMA is a partnership of ESO (representing its member states), NSF (USA) and NINS (Japan), together with NRC (Canada), MOST and ASIAA (Taiwan), and KASI (Republic of Korea), in cooperation with the Republic of Chile. The Joint ALMA Observatory is operated by ESO, AUI/NRAO and NAOJ. MB and EP acknowledge financial support from ASI and INAF under the contract 2017-14-H.0 ASI-INAF. RM and AF acknowledge ERC Advanced Grant 695671 "QUENCH" and support by the Science and Technology Facilities Council (STFC). FF and EP acknowledge financial support from INAF under the contract PRIN INAF 2016 FORECAST.

\section{References}

Aalto, S., Garcia-Burillo, S., Muller, S., et al. 2012, A\&A, 537, A44 Aalto, S., Garcia-Burillo, S., Muller, S., et al. 2015, A\&A, 574, A85 Bischetti, M., Piconcelli, E., Vietri, G., et al. 2017, A\&A, 598, A122 Bourne, M. A., Nayakshin, S., \& Hobbs, A. 2014, MNRAS, 441, 3055 Bourne, M. A., Zubovas, K., \& Nayakshin, S. 2015, MNRAS, 453, 1829 Brusa, M., Cresci, G., Daddi, E., et al. 2018, A\&A, 612, A29 Carilli, C. L., \& Walter, F. 2013, ARA\&A, 51, 105 Carniani, S., Marconi, A., Biggs, A., et al. 2013, A\&A, 559, A29 Carniani, S., Marconi, A., Maiolino, R., et al. 2017, A\&A, 605, A105 Carniani, S., Maiolino, R., Amorin, R., et al. 2018, MNRAS, 478, 1170 Cicone, C., Maiolino, R., Sturm, E., et al. 2014, A\&A, 562, A21 Cicone, C., Maiolino, R., Gallerani, S., et al. 2015, A\&A, 574, A14 Cimatti, A., Daddi, E., Renzini, A., et al. 2004, Nature, 430, 184 Costa, T., Sijacki, D., \& Haehnelt, M. G. 2015, MNRAS, 448, L30 Costa, T., Rosdahl, J., Sijacki, D., \& Haehnelt, M. G. 2018, MNRAS, 479, 2079
Decarli, R., Walter, F., Venemans, B. P., et al. 2017, Nature, 545, 457 Decarli, R., Walter, F., Venemans, B. P., et al. 2018, ApJ, 854, 97 De Looze, I., Baes, M., Bendo, G. J., Cortese, L., \& Fritz, J. 2011, MNRAS, 416, 2712

De Looze, I., Cormier, D., Lebouteiller, V., et al. 2014, A\&A, 568, A62 Díaz-Santos, T., Assef, R. J., Blain, A. W., et al. 2016, ApJ, 816, L6 Di Matteo, T., Springel, V., \& Hernquist, L. 2005, Nature, 433, 604 Fan, L., Knudsen, K. K., Fogasy, J., \& Drouart, G. 2018, ApJ, 856, L5 Faucher-Giguère, C.-A., \& Quataert, E. 2012, MNRAS, 425, 605 Feruglio, C., Fiore, F., Carniani, S., et al. 2015, A\&A, 583, A99 Feruglio, C., Ferrara, A., Bischetti, M., et al. 2017, A\&A, 608, A30 Fiore, F., Feruglio, C., Shankar, F., et al. 2017, A\&A, 601, A143 Fluetsch, A., Maiolino, R., Carniani, S., et al. 2019, MNRAS, 483, 4586 Fruchter, A. S., \& Hook, R. N. 2002, PASP, 114, 144 Gabor, J. M., \& Bournaud, F. 2014, MNRAS, 441, 1615

Gullberg, B., De Breuck, C., Vieira, J. D., et al. 2015, MNRAS, 449, 2883 Hailey-Dunsheath, S., Nikola, T., Stacey, G. J., et al. 2010, ApJ, 714, L162 Herrera-Camus, R., Bolatto, A. D., Wolfire, M. G., et al. 2015, ApJ, 800, 1 Hopkins, P. F., Hernquist, L., Cox, T. J., \& Kereš, D. 2008, ApJS, 175, 356 Ishibashi, W., Fabian, A. C., \& Maiolino, R. 2018, MNRAS, 476, 512 Janssen, A. W., Christopher, N., Sturm, E., et al. 2016, ApJ, 822, 43 Kennicutt, R. C., \& Evans, N. J. 2012, ARA\&A, 50, 531 Kimball, A. E., Lacy, M., Lonsdale, C. J., \& Macquart, J.-P. 2015, MNRAS, 452, 88

Kormendy, J., \& Ho, L. C. 2013, ARA\&A, 51, 511

Longinotti, A. L., Vega, O., Krongold, Y., et al. 2018, ApJ, 867, L11

Maiolino, R., Cox, P., Caselli, P., et al. 2005, A\&A, 440, L51

Maiolino, R., Gallerani, S., Neri, R., et al. 2012, MNRAS, 425, L66

McMullin, J. P., Waters, B., Schiebel, D., Young, W., \& Golap, K. 2007, in

Astronomical Data Analysis Software and Systems XVI, eds. R. A. Shaw, F. Hill, \& D. J. Bell, ASP Conf. Ser., 376, 127

Menci, N., Fiore, F., Puccetti, S., \& Cavaliere, A. 2008, ApJ, 686, 219

Neri, R., Downes, D., Cox, P., \& Walter, F. 2014, A\&A, 562, A35

Papadopoulos, P. P., van der Werf, P., Isaak, K., \& Xilouris, E. M. 2010, ApJ, 715, 775

Peng, Y., Maiolino, R., \& Cochrane, R. 2015, Nature, 521, 192

Polletta, M., Tajer, M., Maraschi, L., et al. 2007, ApJ, 663, 81

Richings, A. J., \& Faucher-Giguère, C.-A. 2018, MNRAS, 474, 3673

Roos, O., Juneau, S., Bournaud, F., \& Gabor, J. M. 2015, ApJ, 800, 19

Runnoe, J. C., Brotherton, M. S., \& Shang, Z. 2012, MNRAS, 422, 478

Rupke, D. S., Veilleux, S., \& Sanders, D. B. 2005, ApJS, 160, 115

Sargsyan, L., Lebouteiller, V., Weedman, D., et al. 2012, ApJ, 755, 171

Stacey, G. J., Hailey-Dunsheath, S., Ferkinhoff, C., et al. 2010, ApJ, 724, 957

Straatman, C. M. S., Labbé, I., Spitler, L. R., et al. 2014, ApJ, 783, L14

Tombesi, F., Meléndez, M., Veilleux, S., et al. 2015, Nature, 519, 436

Trakhtenbrot, B., Lira, P., Netzer, H., et al. 2017, ApJ, 836, 8

Vayner, A., Wright, S. A., Murray, N., et al. 2017, ApJ, 851, 126

Veilleux, S., Bolatto, A., Tombesi, F., et al. 2017, ApJ, 843, 18

Venemans, B. P., Walter, F., Zschaechner, L., et al. 2016, ApJ, 816, 37

Venemans, B. P., Walter, F., Decarli, R., et al. 2017, ApJ, 837, 146

Wang, R., Wagg, J., Carilli, C. L., et al. 2013, ApJ, 773, 44

Wang, R., Wu, X.-B., Neri, R., et al. 2016, ApJ, 830, 53

Whitaker, K. E., van Dokkum, P. G., Brammer, G., et al. 2013, ApJ, 770, L39

Willott, C. J., Omont, A., \& Bergeron, J. 2013, ApJ, 770, 13

Willott, C. J., Bergeron, J., \& Omont, A. 2015, ApJ, 801, 123

Willott, C. J., Bergeron, J., \& Omont, A. 2017, ApJ, 850, 108

Zubovas, K., \& King, A. 2012, ApJ, 745, L34 


\section{Appendix A: Spectral fitting}

In order to derive the parameters of the [CII] core and broad [CII] wings emission, we performed spectral fitting of the stacked spectra through $\chi^{2}$ minimisation, by using the python scipy optimize package curve_fit. We used a fitting model that includes one or two Gaussian components to reproduce the [CII] core plus an additional broad Gaussian component to account for the highvelocity $[\mathrm{CII}]$ wings. All parameters were left free to vary in the fits with no constrains. In Fig. A.1, we show the confidence ellipses associated with the parameters of the broad Gaussian component modelling the [CII] wings. Despite the correlation between the line width and the normalisation of the wings, the free outflow parameters remain well constrained.

In addition to the significance estimated through the spectral fitting analysis, that is from the errors on the best-fit parameters, we provided additional significance estimates by considering the pure statistical uncertainties of the stacked spectra. After removing the modelled [CII] core emission from the stacked spectra, we calculated the integrated flux associated with the broad component alone. We therefore computed the statistical uncertainty of the ALMA map integrated over the same spectral interval. The statistical significance can be therefore derived as the ratio between the integrated flux and the statistical uncertainty. This estimate results in a higher confidence than that estimated from the fit, as it does not take into account the uncertainties in modelling the [CII] core. Moreover, to estimate the significance of the high-velocity channels alone, we repeated the same procedure but excluding the velocity range covered by the [CII] core.

In Fig. A.2, we also show the best fit 1-Gaussian component model for each stacked spectrum. In almost all panels, although with different significance levels, emission in excess of a single Gaussian component is present.
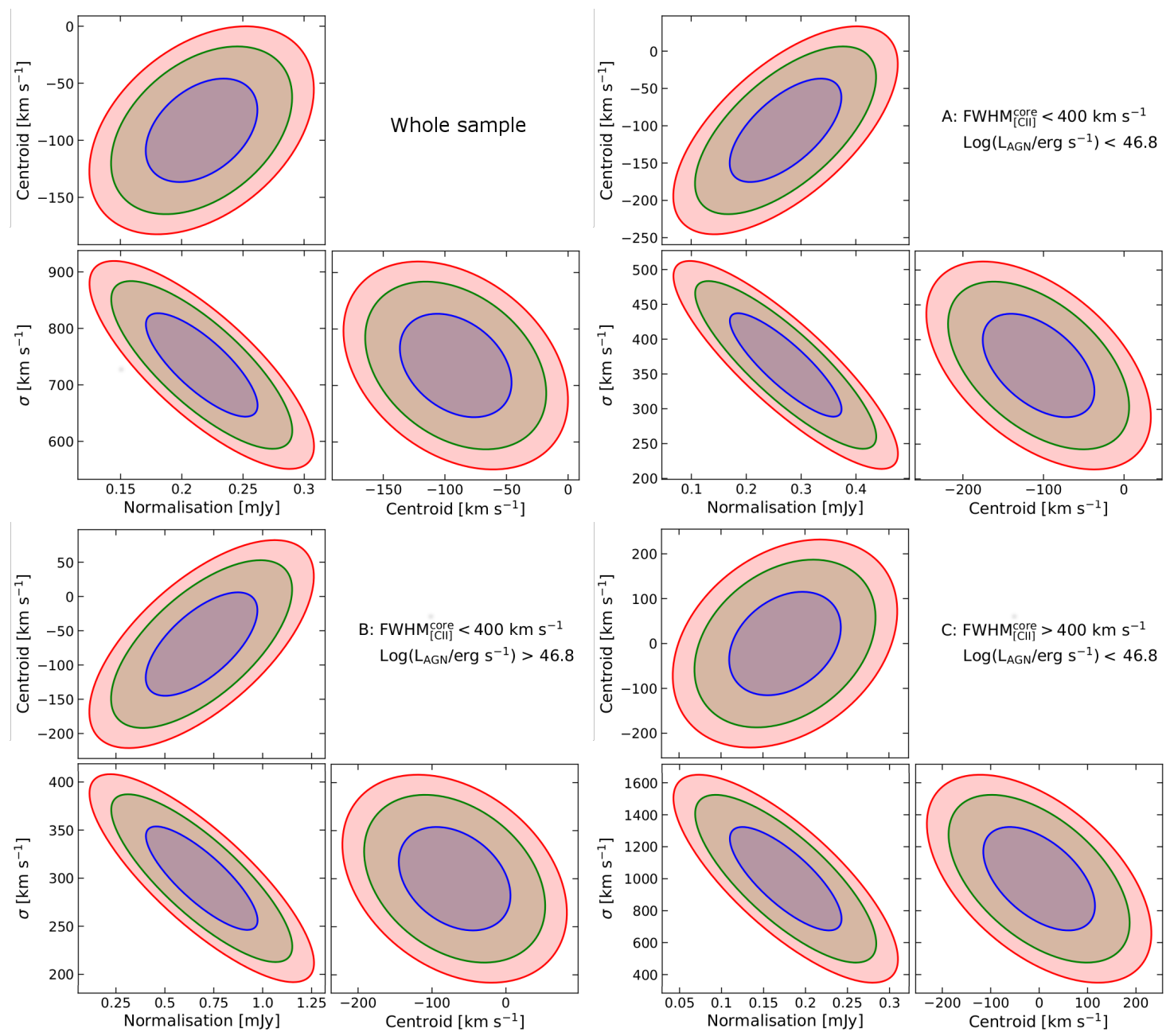

Fig. A.1. Confidence ellipses for the parameters of the broad Gaussian component modelling the [CII] wings. For each stacked spectrum (indicated by the top label) the $1 \sigma, 2 \sigma$ and $3 \sigma$ confidence intervals are indicated by the blue, green and red ellipses, respectively. 
M. Bischetti et al.: Widespread QSO-driven outflows in the early Universe
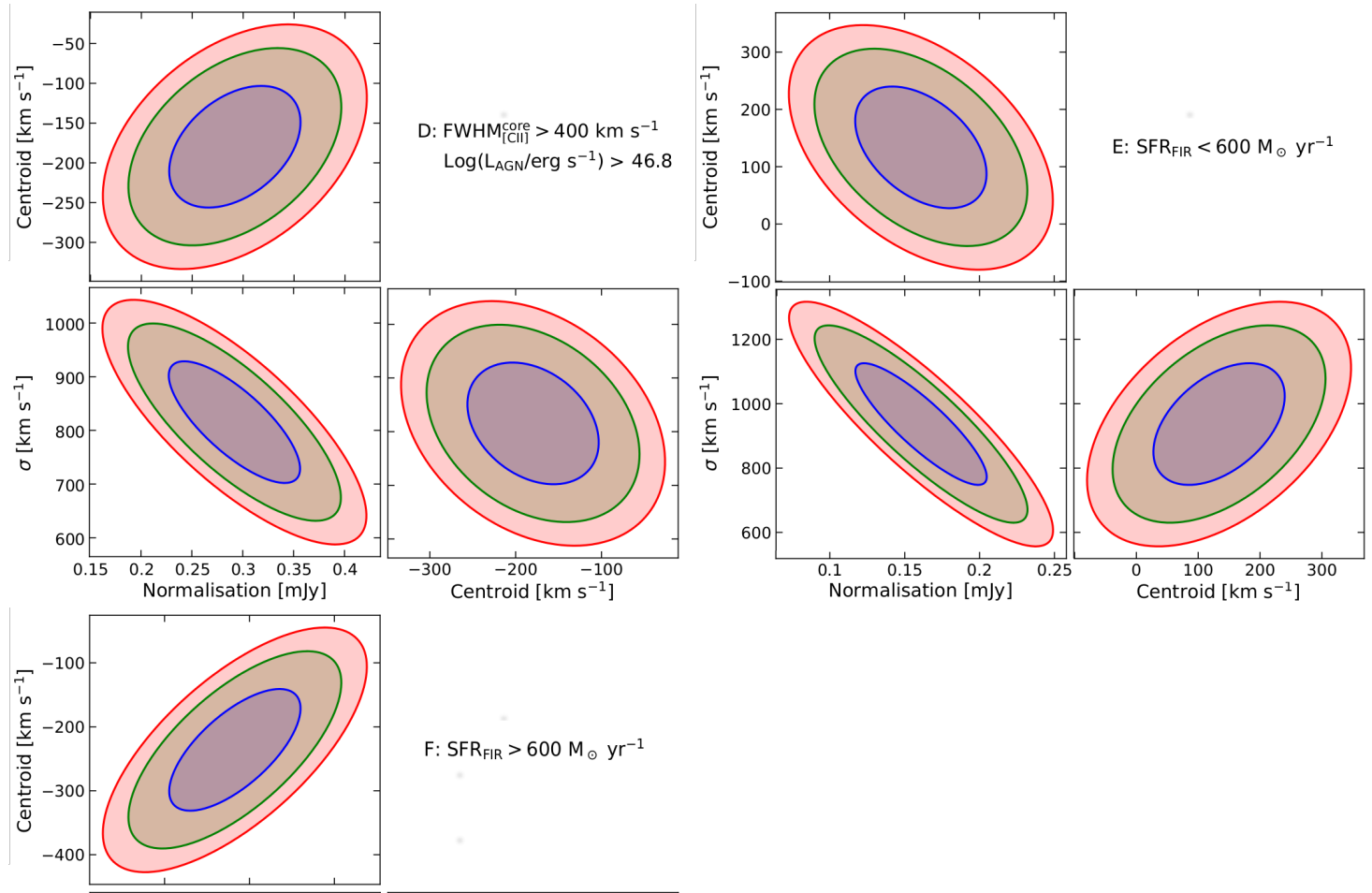

$\mathrm{F}: \mathrm{SFR}_{\mathrm{FIR}}>600 \mathrm{M}_{\odot} \mathrm{yr}^{-1}$

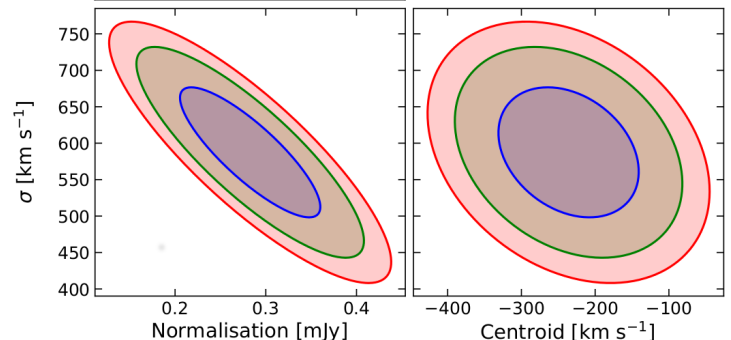

Fig. A.1. continued.
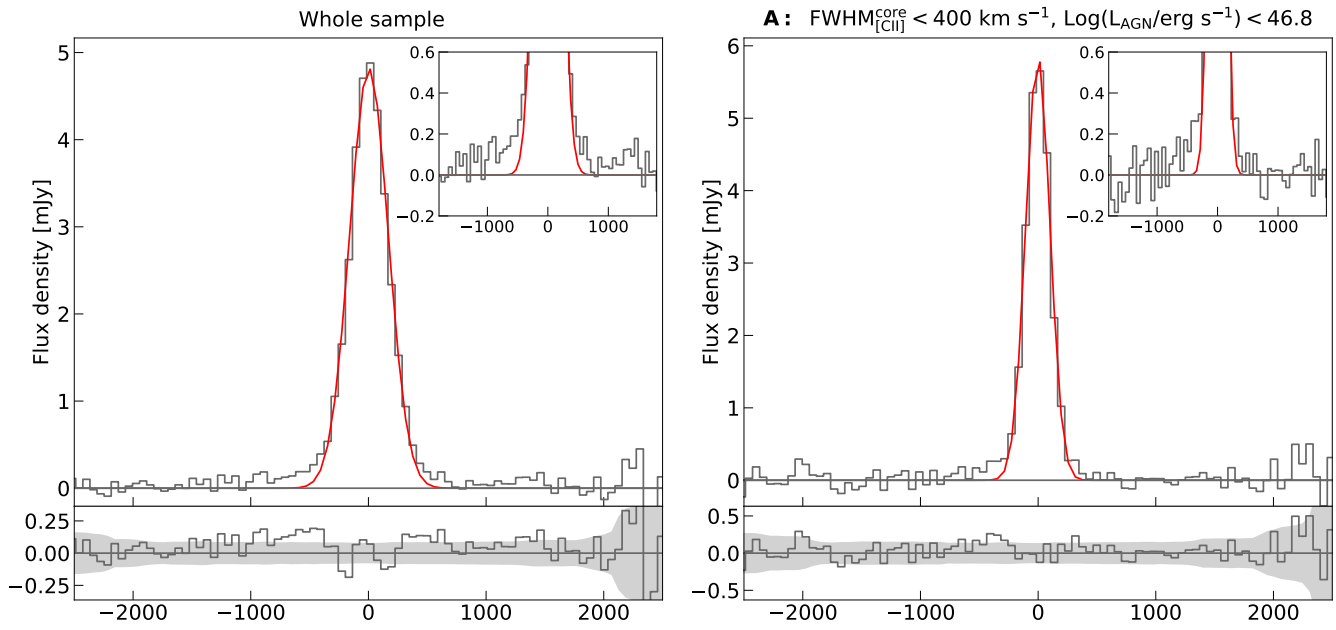

Fig. A.2. Stacked integrated spectra for the whole sample and all the QSOs subgroups presented in Sect. 4.4 and Sect. 4.2. For each plot, the top panel shows the [CII] flux density as a function of velocity, in bins of $60 \mathrm{~km} \mathrm{~s}^{-1}$. The red curve represents the best-fit 1 Gaussian component model. The inset zooms on the $v \in[-1500,1500] \mathrm{km} \mathrm{s}^{-1}$ region, while the bottom panel shows the residuals at different velocities. The $1 \sigma \mathrm{rms}$ of the spectrum is also indicated by the shaded region. 
B : $\mathrm{FWHM}_{[\mathrm{CIII}}^{\text {core }}<400 \mathrm{~km} \mathrm{~s}^{-1}, \log \left(\mathrm{L}_{\mathrm{AGN}} / \mathrm{erg} \mathrm{s}^{-1}\right)>46.8$

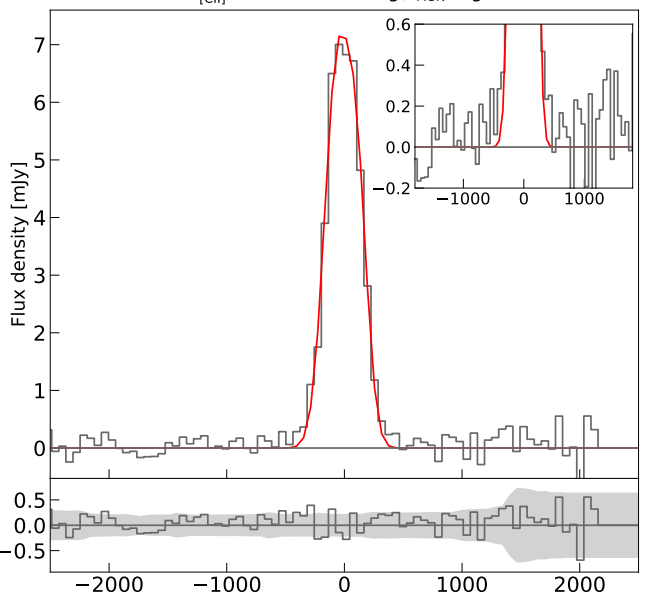

D : $\quad F_{W H M}^{\text {core }}>400 \mathrm{~km} \mathrm{~s}^{-1}, \log \left(L_{\text {AGN }} / \mathrm{erg} \mathrm{s}^{-1}\right)>46.8$

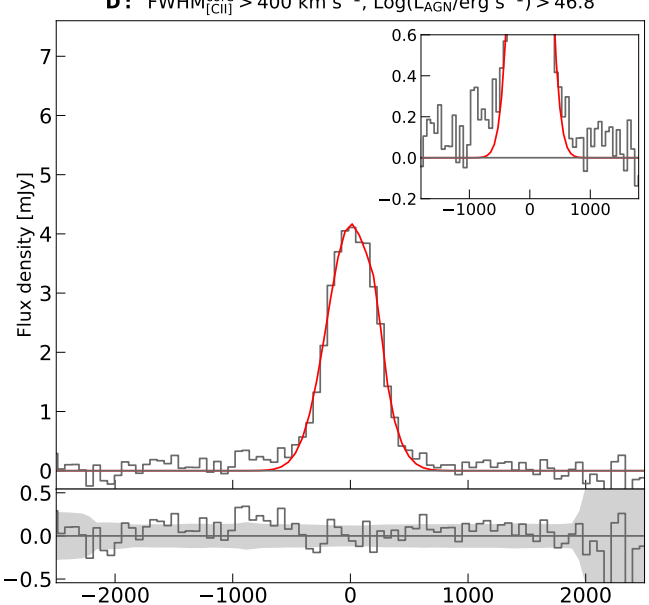

C: $\quad$ FWHM $_{[C I I]}^{\text {core }}>400 \mathrm{~km} \mathrm{~s}^{-1}$, $\log \left(\mathrm{L}_{\mathrm{AGN}} / \mathrm{erg} \mathrm{s}^{-1}\right)<46.8$

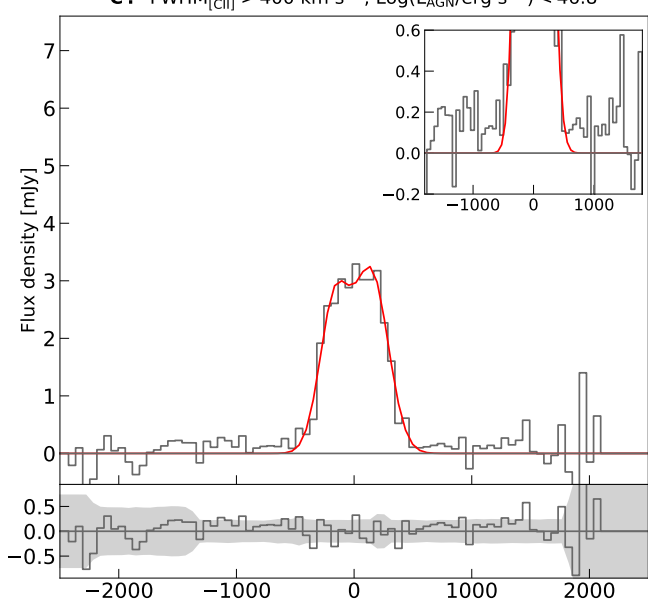

E : $\mathrm{SFR}_{\mathrm{FIR}}<600 \mathrm{M}_{\odot} \mathrm{yr}^{-1}$

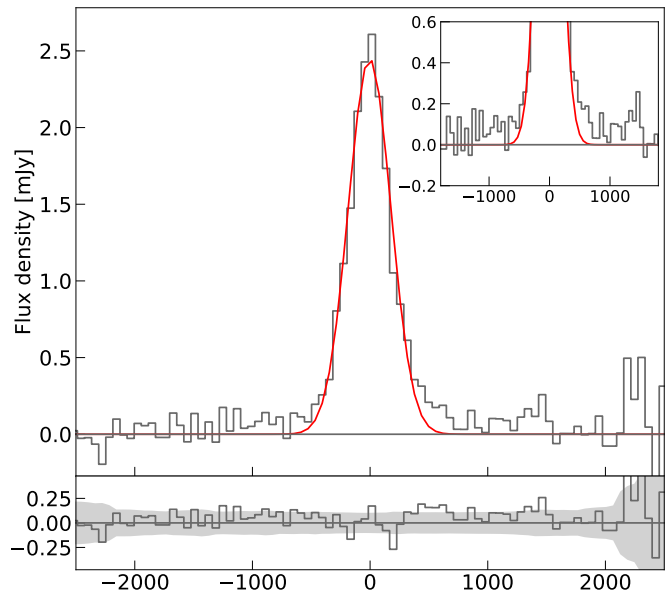

: $\mathrm{SFR}_{\mathrm{FIR}}>600 \mathrm{M}_{\odot} \mathrm{yr}^{-1}$

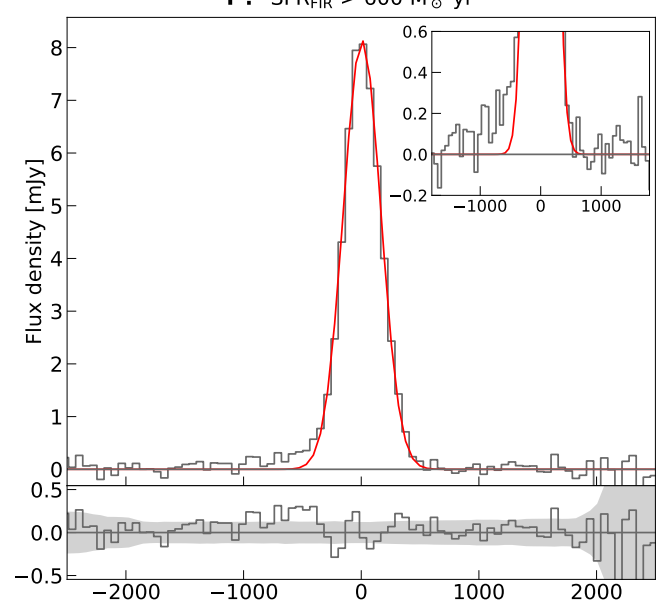

Fig. A.2. continued. 


\section{Appendix B: Stack in luminosity density units}

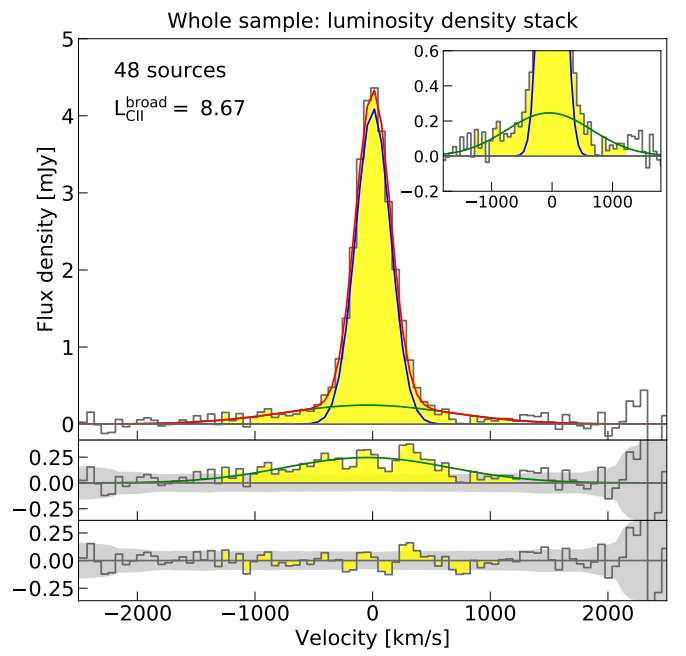

Fig. B.1. Whole sample integrated spectrum, stacked in luminosity density units. First panel from top: [CII] flux density as a function of velocity, in bins of $60 \mathrm{~km} \mathrm{~s}^{-1}$. The red curve represents the best-fit 2 Gaussian components model; the two individual components are shown with blue and green curves. Labels indicate the number of stacked sources and the luminosity of the broad [CII] wings. The inset shows a zoom on the broad component. Second panel from top: residuals from the subtraction of the core emission (blue line in first panel). The green curve shows the best fit broad component. Third panel: residuals from the two Gaussian components fitting. The $1 \sigma \mathrm{rms}$ of the spectrum is also indicated by the shaded region.

The QSOs in our sample span the redshift interval $4.5 \lesssim z \lesssim 7$ and, therefore, a factor of 1.7 in luminosity distance. Accordingly, we verified that our results are still valid if performing a stack in luminosity density units. We used the $z_{[\mathrm{CII}]}$-based (see Table 1) luminosity distance to convert each spectrum in luminosity units and then performed the stack of the whole sample by using Eq. (1) and (2). By applying the same fitting procedure presented in Sect. 4.1, we derived a luminosity of the broad [CII] wings of $L_{[\mathrm{CIII}]}^{\text {broad }} \sim 4.7 \times 10^{8} L_{\odot}$ (see Fig. B.1), similar to the value estimated from the original stack in flux density units (reported in Table 2).

\section{Appendix C: Stack over same physical scales}

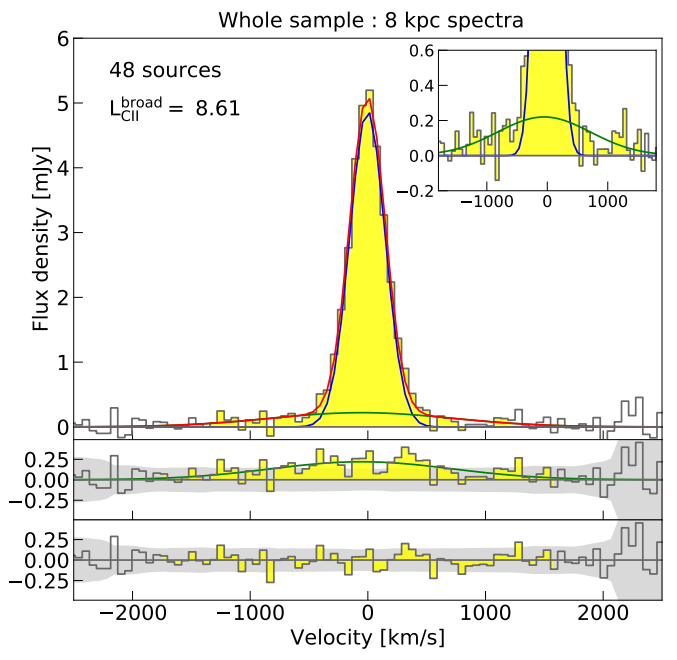

Fig. C.1. Whole sample integrated spectrum, obtained by stacking spectra of the inner $8 \mathrm{kpc}$ around the QSOs in our sample. Panels are as in Fig. B.1.

The ALMA observations of the QSOs in our sample are characterised by different angular resolutions, with sizes of the ALMA beam in the range $\sim 0.4-1.2$ arcsec. This implies that emission from different spatial scales may contribute to the stacked spectrum of the total sample (Sect. 4.1). To ensure that broad [CII] wings persist when combining spectra from the same physical scales around the QSO, we extracted spectra of all QSOs in our sample from a circular aperture with radius equal to $8 \mathrm{kpc}$, that is the largest scale probed by our observations (Table 1). We then stacked them by following the procedure presented in Sect. 3. Fig. C. 1 shows that, although the increased noise fluctuations in the stacked spectrum, associated with the chosen large extraction aperture size, the [CII] wings are still present and characterised by a luminosity $L_{[\mathrm{CII}]}^{\text {broad }} \sim 4 \times 10^{8} L_{\odot}$, close to the value estimated from the original stack in Sect. 4.1. 